\title{
Management of tooth resorption
}

\author{
GS Heithersay*
}

\begin{abstract}
A correct diagnosis and an understanding of the aetiology and dynamics of the processes involved in tooth resorption is critical to effective management. Tooth resorptions can be classified as: (1) trauma induced; (2) infection induced; or (3) hyperplastic invasive. Some transient trauma induced resorptions require no treatment but must be carefully monitored to check that there are no complicating issues such as infection. In cases of trauma induced replacement resorption, a multidisciplinary approach is usually necessary to ensure an optimal long-term solution. Infection induced tooth resorptions require the removal of the invading micro-organisms by endodontic therapy including intra-canal medication which can also facilitate repair of the resorbed tooth structure. The hyperplastic invasive tooth resorptions pose considerable challenges in management due to the complexity and aggressive nature of the resorptive process. With careful case selection and complete inactivation of resorptive tissue successful management can be achieved.
\end{abstract}

Key words: Tooth resorption, endodontics, dental trauma, hyperplastic tooth resorptions.

\section{INTRODUCTION}

Dental clinicians can be faced with difficult diagnostic and treatment decisions with respect to tooth resorption. Tooth resorption in the primary and permanent dentition has been extensively studied and the complex processes involved in the removal of the organic and inorganic components of tooth structure by clastic cells continue to evolve through basic research (for reviews see Pierce, ${ }^{1}$ Dreyer et al., ${ }^{2}$ Davidovitch et al. $\left.{ }^{3}\right)$. Knowledge gained from experimental studies and observations of histopathological material has provided a sound basis for the diagnosis and treatment of many tooth resorptive processes. ${ }^{4,5}$

The aim of this paper is to simplify for general dental practitioners the diagnosis and clinical management of tooth resorption by focusing on those resorptions which do not and those which do require treatment, and to identify other resorptions where combined or alternative treatment is indicated. Emphasis will be placed on preventive measures to control resorption,

\footnotetext{
*Clinical Professor, Endodontic Teaching Group, School of Dentistry, Faculty of Health Sciences, The University of Adelaide, South Australia.
}

particularly following luxation and avulsion injuries. These important preventive measures rely on a recognition of healing responses which are possible in each particular clinical situation. It is the legacy of the research of Andreasen and co-workers that responses to a variety of traumatic injuries have been so thoroughly analysed as to make the management of dental injuries predictable and usually highly successful. The latest edition of Textbook and Color Atlas of Traumatic Injuries to the Teeth, ${ }^{6}$ or Traumatic Dental Injuries; A Manual are highly recommended for all dental practitioners. Successful management of potential or established resorption in a dentally traumatized patient is of paramount importance, as it has been recognized that poorly treated injuries can have not only physical consequences but also a significant psychological impact on the patient.

When a patient presents with tooth resorption, the following basic questions must be addressed in arriving at a diagnosis and treatment plan:

(1) What type of resorption is present?

(2) Is the resorption external (periodontally derived), internal (pulpally derived) or communicating?

(3) Will the resorptive process be self-limiting or transient and not require management other than careful monitoring of healing processes?

(4) If the resorptive process is progressive will there be a favourable response to treatment and, if so, what is the appropriate therapy?

(5) If treated what are the short and long-term prognoses?

(6) When is extraction and prosthetic therapy indicated?

\section{Classification of dental resorptions}

Classifications play an important role for the clinician in the process of diagnosis and treatment planning. Andreasen has over the past 40 years made an unique contribution to the understanding of tooth resorption following dental trauma and his original classification which follows (Fig 1) remains the most widely accepted. ${ }^{8}$ However, Andreasen's original classification does not include other resorptive processes which have been identified over the past two decades. Of these, a third type of internal resorption, transient apical internal surface resorption, ${ }^{9}$ should also 


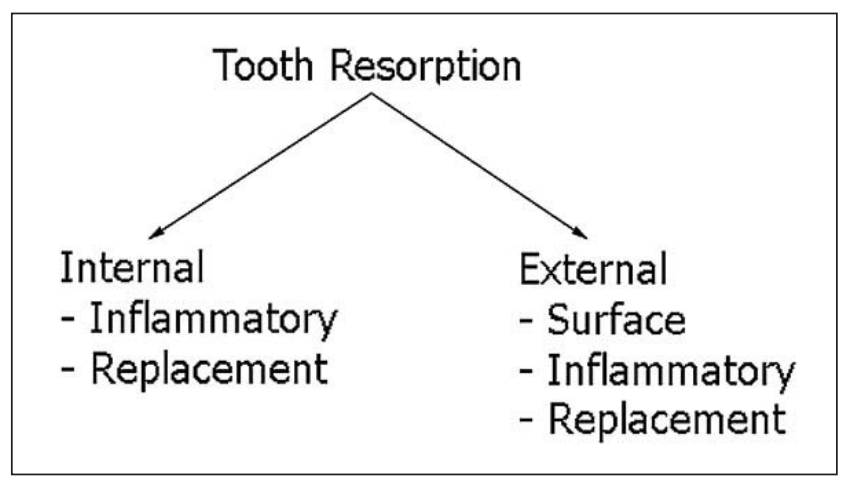

Fig 1. Andreasen classification of tooth resorption.

be added, along with other types of hyperplastic tooth resorption..$^{10}$ These hyperplastic resorptions - labelled invasive coronal, invasive cervical or invasive radicular resorption - do not fall into any of the original categories but may follow dental trauma and other potential predisposing factors. ${ }^{11}$

An alternative classification of tooth resorption recently proposed by Lindskog ${ }^{12}$ will be adopted for this paper. This classification subdivides resorptions into three broad groups: (1) trauma induced tooth resorption; (2) infection induced tooth resorption; and (3) hyperplastic invasive tooth resorptions.

There are some rare tooth resorptions of unknown cause that do not fit into any of the above categories and they are usually labelled "idiopathic". The advantage of this classification is that it makes a simple and clear distinction between each category and as such provides important clues to the clinical management. For example, infection induced tooth resorption as with any infective process requires elimination of invading micro-organisms as an integral component of clinical management.

\section{Trauma induced tooth resorption}

In this category using a broader interpretation of the term "trauma", resorptions may have resulted from pressure from unerupted or erupting teeth or some neoplasms, from biomechanical forces involved in orthodontics, mechanical trauma (luxation and avulsion injuries) and surgical, thermal or chemical trauma. In all trauma induced (non-infective) tooth resorption some damage to the cementum/cementoid-periodontal membrane complex has occurred which stimulates clastic activity. Trauma induced tooth resorption may be subdivided into: (1) surface resorption; (2) transient apical internal resorption; (3) pressure resorption; (4) orthodontic resorption; and (5) replacement resorption.

\section{Surface resorption}

From a treatment management point of view, the simplest form of trauma induced (non-infective) resorption is surface resorption which, as the name suggests, is a shallow resorption of cementum often with involvement of a small amount of underlying dentine. This type of resorption is self-limiting and

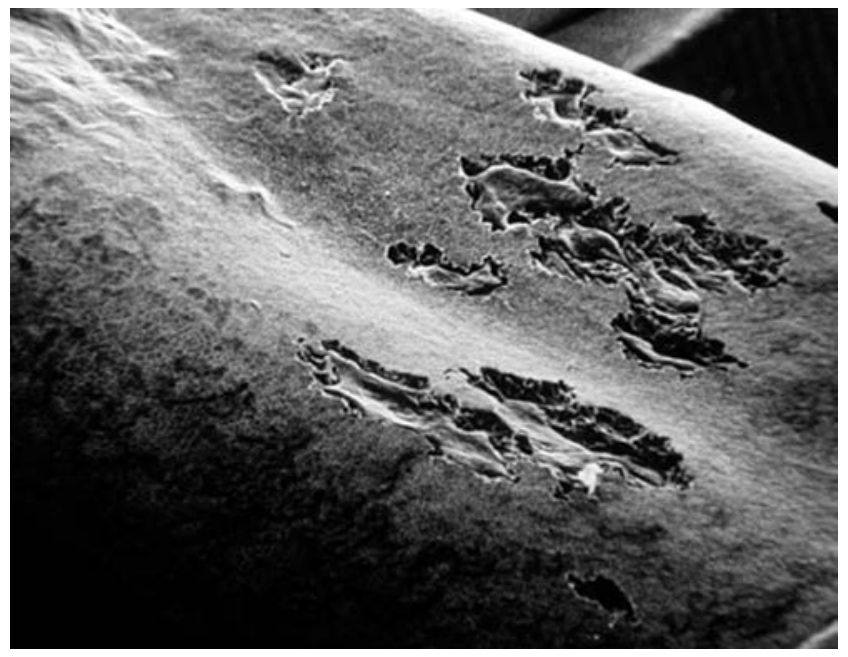

Fig 2. Photomicrograph of a tooth displaying trauma induced surface resorption. Courtesy Prof S Lindskog.

transient and can follow some traumatic injuries or orthodontic treatment. In the absence of superimposed infection, usually in the root canal, surface resorption as shown in the scanning electron micrograph in Fig 2 will heal uneventfully with reparative cementum as shown histologically in Fig 3. Clinically, surface resorption may be difficult to observe radiographically as there may be no or only slight changes in the root morphology and the image of the periodontal membrane and associated lamina dura remains intact with no signs of any associated root or bony radiolucency. An example of the slight change in root contour following healing of surface resorption is demonstrated in

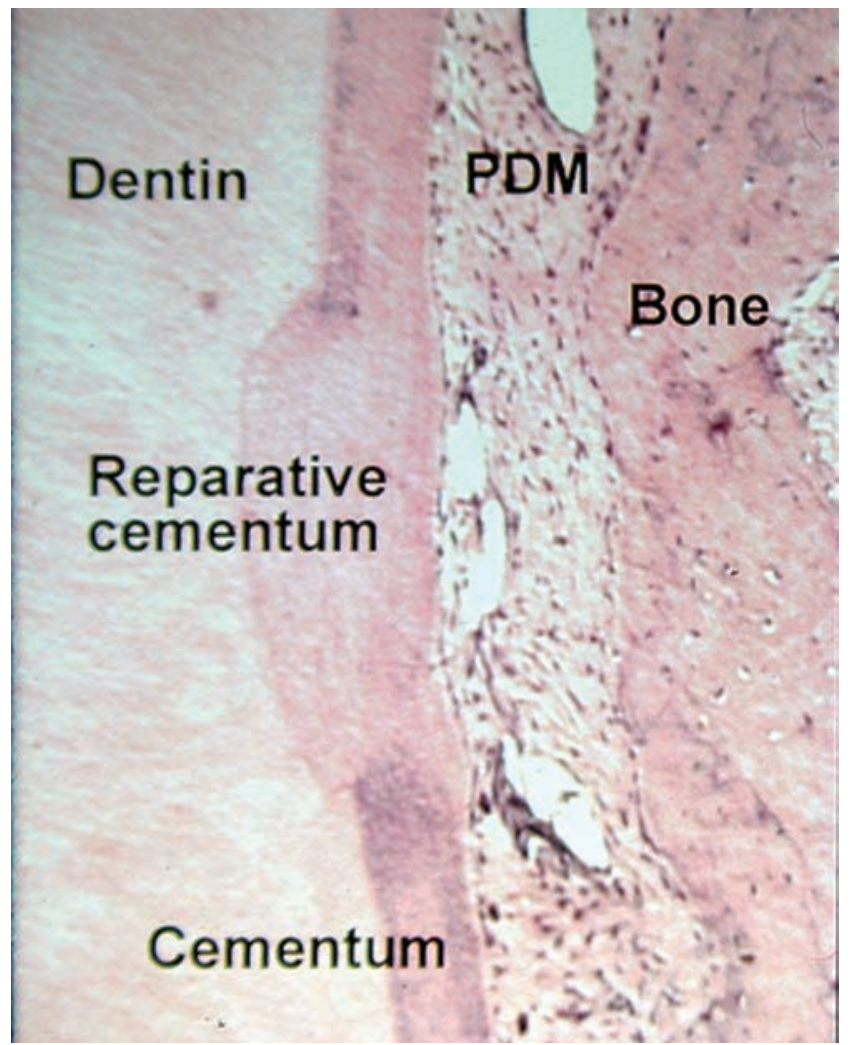

Fig 3. Histological appearance of repaired trauma induced surface resorption showing reparative cementum. Courtesy Prof S Lindskog. 

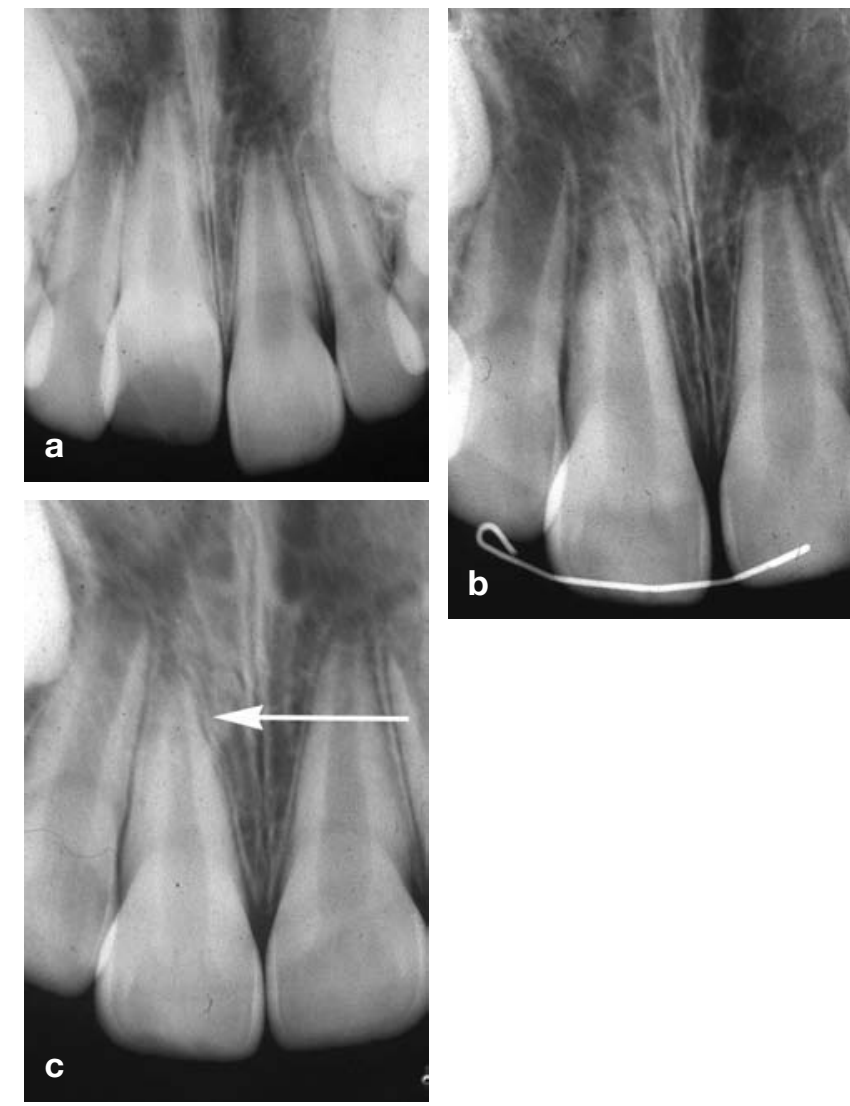

Fig 4a. Radiograph showing evidence of intrusion of the maxillary right central incisor of an eight year old female. The intruded tooth was surgically repositioned within 30 minutes of the injury.

Fig 4b. Radiograph taken 18 days after surgical repositioning of the intruded central incisor.

Fig 4c. Six months later a slight change in root contour is evident on the mesial aspect of the repositioned tooth (arrow) indicative of trauma induced surface resorption.

Fig 4 which shows a maxillary right central incisor of an eight year old female which was repositioned within 30 minutes of its intrusion. Because of the immature root development the tooth was not endodontically treated but monitored closely for evidence of revascularization which was demonstrated by progressive root canal narrowing due to reparative dentine deposition.

\section{Transient apical internal resorption}

Transient apical internal resorption is another form of trauma induced non-infective root resorption which was identified by Andreasen in $1986 .^{9}$ This resorptive process can follow luxation injuries and may be associated with a transient apical breakdown recognized by a confined periapical radiolucency which resolves within a few months. It is considered to be a positive response, with the internal apical resorption allowing ingress of a greater vascular network to aid in the healing of a traumatized pulp. Often there is an associated colour change due to intra-pulpal haemorrhage and this may resolve spontaneously if revasularization to the coronal pulp chamber occurs. In the longer term, as this is a transient process, the internally resorbed apex will close uneventfully.
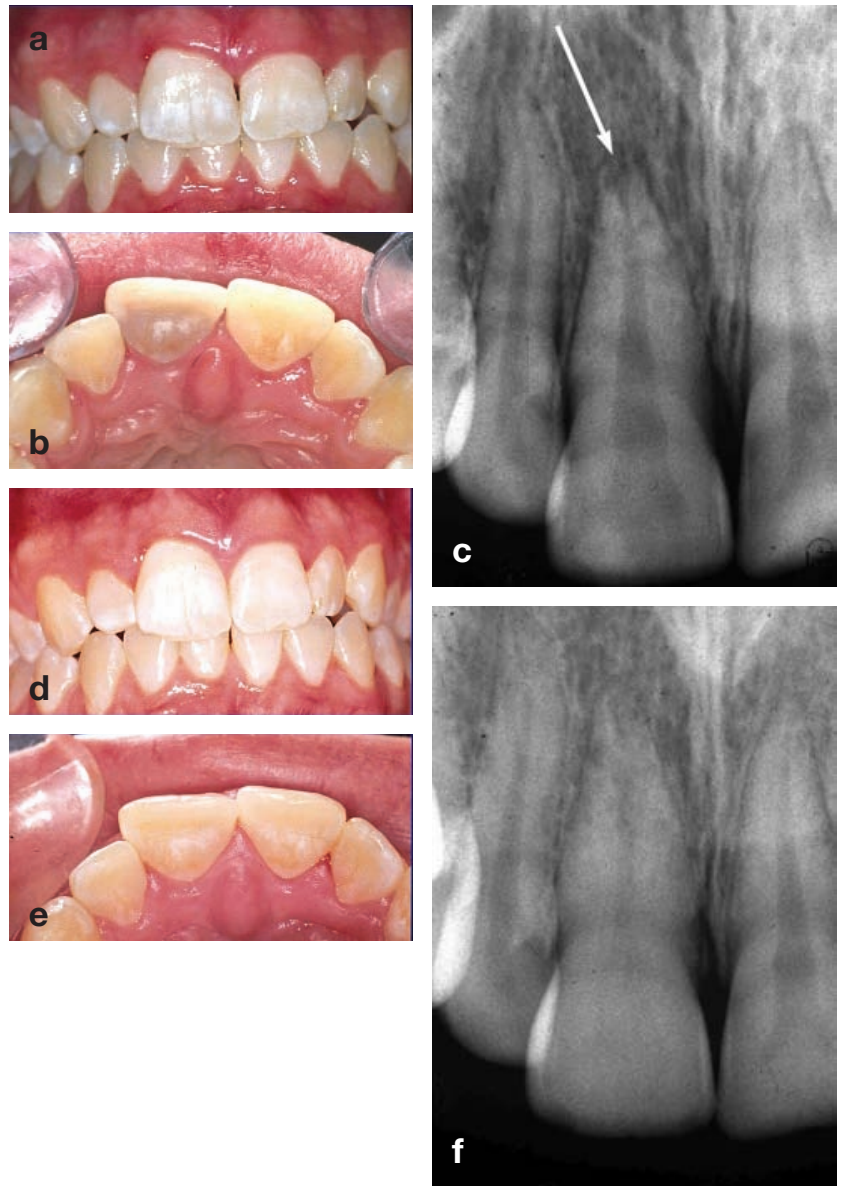

Fig 5a. Labial view of a maxillary right central incisor which had been palatally luxated and repositioned 1 month earlier showing slight coronal discolouration.

Fig $5 \mathrm{~b}$. The palatal view reveals more extensive discolouration in the traumatized maxillary right central incisor.

Fig 5c. Radiograph taken 1 month after the luxation injury shows evidence of transient apical internal resorption (arrow)

Fig 5 d. One year later there has been total restitution of tooth colour.

Fig 5e. Palatal view showing colour reversal.

Fig $5 \mathrm{f}$. Radiograph taken 1 year after the original trauma shows resolution of the apical internal resorption and no other signs of periradicular pathosis.

An example of transient apical internal root resorption is shown in Fig 5. This case illustrates the resorptive process following a luxation injury, tooth discolouration and resolution, and then apical closure. The resolution of discolouration which may accompany transient apical internal resorption does not occur in all cases. In the experience of the author it is estimated that colour resolution will occur in approximately 50 per cent of cases despite other radiographic signs of a satisfactory healing response. This poses an aesthetic problem which on occasions may be resolved by external bleaching, but more often elective pulpectomy, root filling and intra-coronal bleaching are required. If internal apical resorption is still present, the endodontic treatment is somewhat complicated and should extend only to the position of the resorption. These aesthetic complications and the difficulty posed for dental practitioners in monitoring this healing response have led some to question the advisability of recommending this conservative approach as opposed to pulpectomy, 


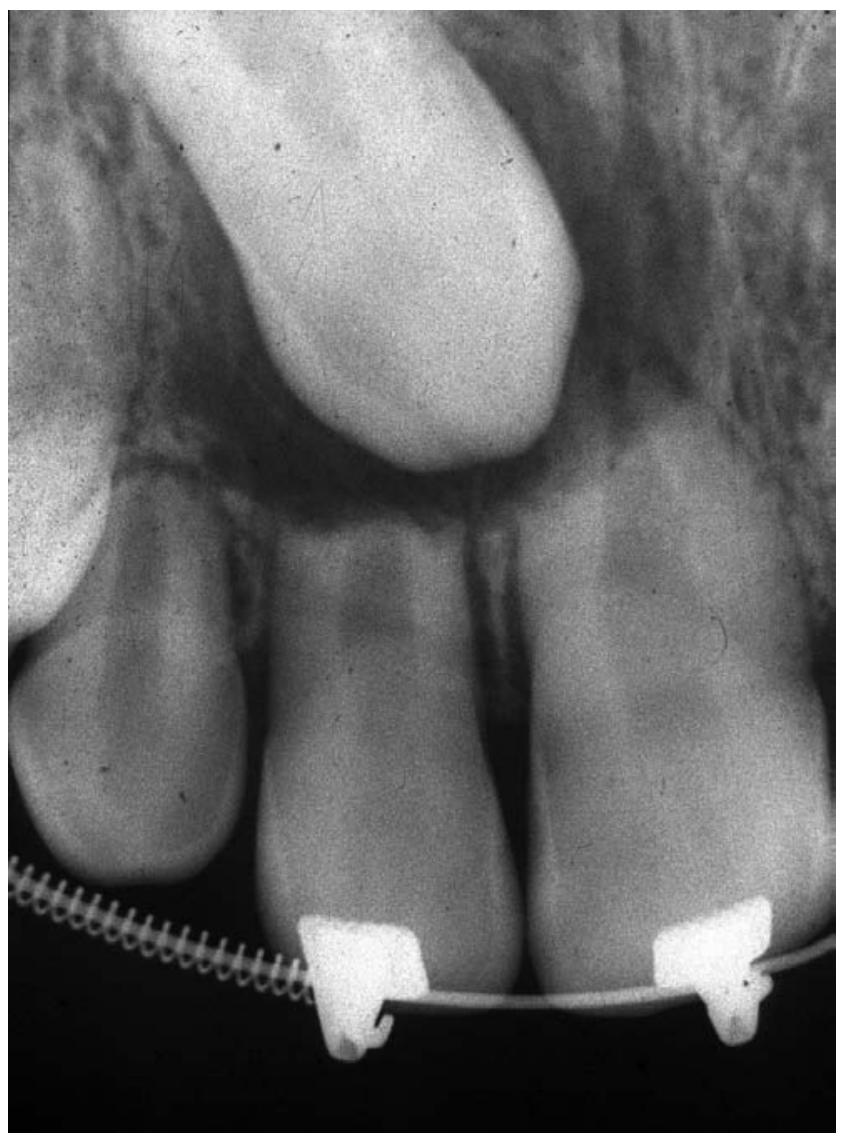

Fig 6. Extensive trauma induced pressure resorption is evident in the radiograph of the maxillary right central and lateral incisors due to an unerupted cuspid tooth and its crypt.

root filling and intra-coronal bleaching as soon as discolouration is observed. However, the author remains of the opinion that this healing process should be recognized and implemented in clinical practice and teaching.

\section{Pressure resorption and orthodontic resorption}

More extensive trauma induced non-infective root resorption may be induced by the pressure of a crypt of an unerupted/erupting tooth or some neoplasms and more commonly during orthodontic treatment. The resorption is often extensive and easily observable radiographically. Figure 6 shows the radiographic appearance of extensive resorption of a maxillary central and lateral incisor due to pressure from an unerupted cuspid and an example of significant root resorption following orthodontic treatment is shown in shown in Fig 7. With the removal of the initiating "trauma", these non-infective resorptions will become inactive and uncomplicated repair will occur.

\section{Replacement resorption}

The most serious form of trauma induced noninfective root resorption is replacement resorption which, as the name suggests, involves the progressive replacement of tooth structure by alveolar bone and ultimately tooth loss. Replacement resorption follows the death of viable periodontal ligament cells due to

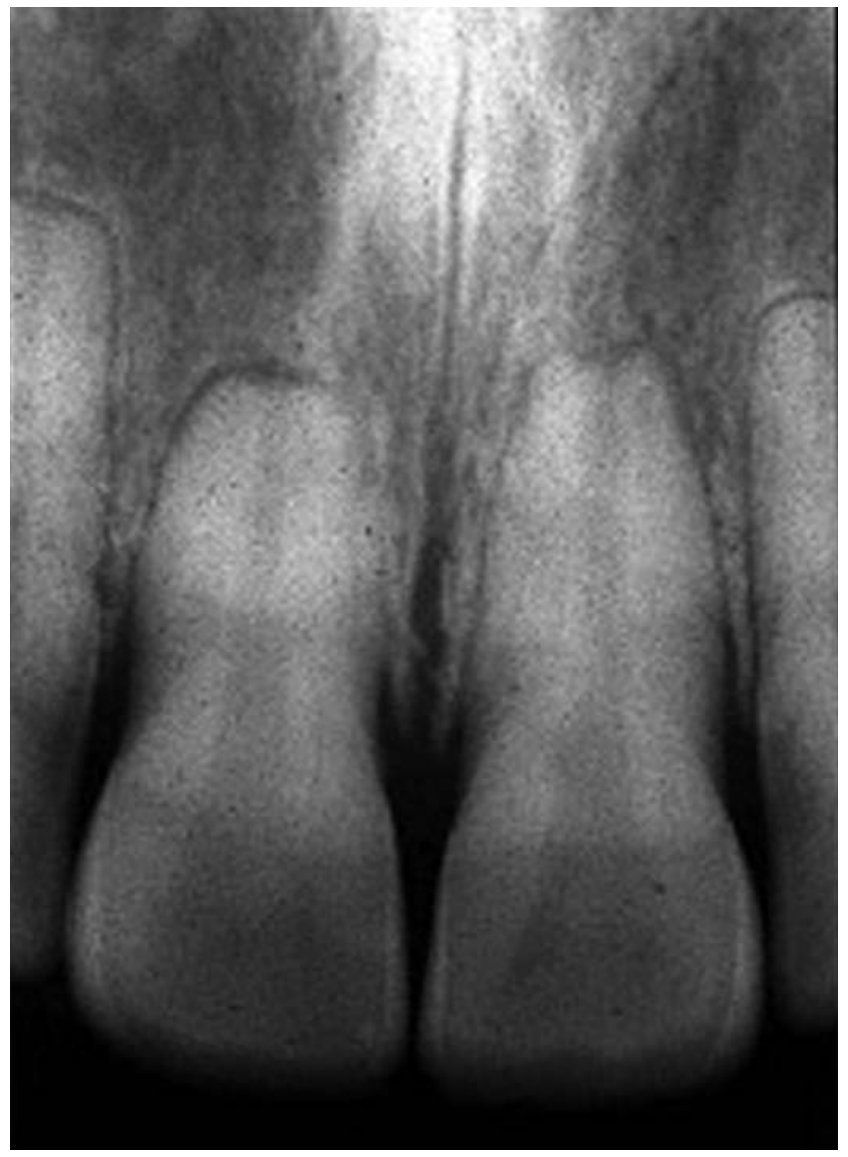

Fig 7. Radiograph showing trauma induced orthodontic root resorption in an 18 year old male.

factors such as compression or drying of the ligament cells as in the case of delayed replantation of an avulsed tooth. On rare occasions an intact cementum/ cementoid layer may act as a biological barrier, so that ankylosis (i.e., union with bone) is not accompanied by replacement resorption. However, the usual response is that of ankylosis with replacement resorption due to the development, subsequent to surface resorption, of an interface between bone and dentine with remodelling processes occurring as part of normal skeletal bone turn-over, but at the expense of dentine. There is total loss of mobility due to this union of tooth and bone, and the tooth gives a characteristically high percussion sound but otherwise patients with replacement resorption are symptom-free. Radiographically there will be total loss of the image of the periodontal ligament followed by evidence of the progressive replacement of tooth structure by bone - in time the image of the tooth root is lost. An example of an ankylosed tooth which is undergoing replacement resorption is shown in Fig 8. At present, there is no treatment possible for this type of resorption and so the clinical management from the initiation of replacement resorption to the inevitable demise of the tooth poses important challenges particularly in the developing dentition.

If a tooth is in a satisfactory position in a mature dentition, there is no urgency for tooth replacement as often the replacement resorption proceeds at a slow 


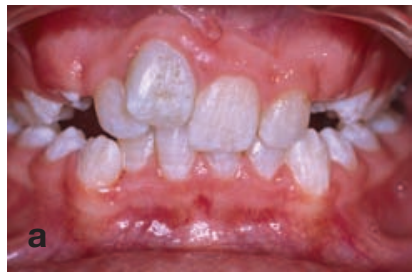

Fig 8a. Labial appearance of an 11 year old female showing the maxillary right central incisor in infra-occlusion as a result of trauma induced replacement resorption.

Fig 8 b. Radiograph of Fig $8 \mathrm{a}$ showing the image of the replacement of tooth structure by bone which characterizes trauma induced replacement

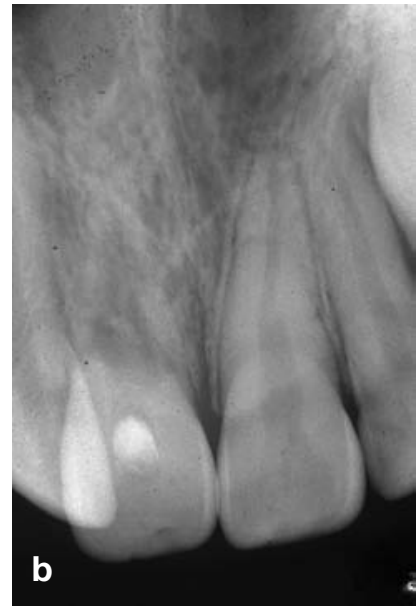
resorption.

rate - in some instances taking many years to reach a stage where carefully planned intervention is necessary. This provides valuable time for both the clinician to plan elective treatment, ideally in the form of implant therapy, and for the patient to prepare himself/herself both mentally and financially for that procedure. An example of a tooth which has been undergoing replacement resorption at a slow rate is shown in Fig 9. In this instance, endodontic therapy had been carried out 13 years earlier to reduce the possibility of superimposed infection, but such endodontic intervention has no effect on the progression of replacement resorption.

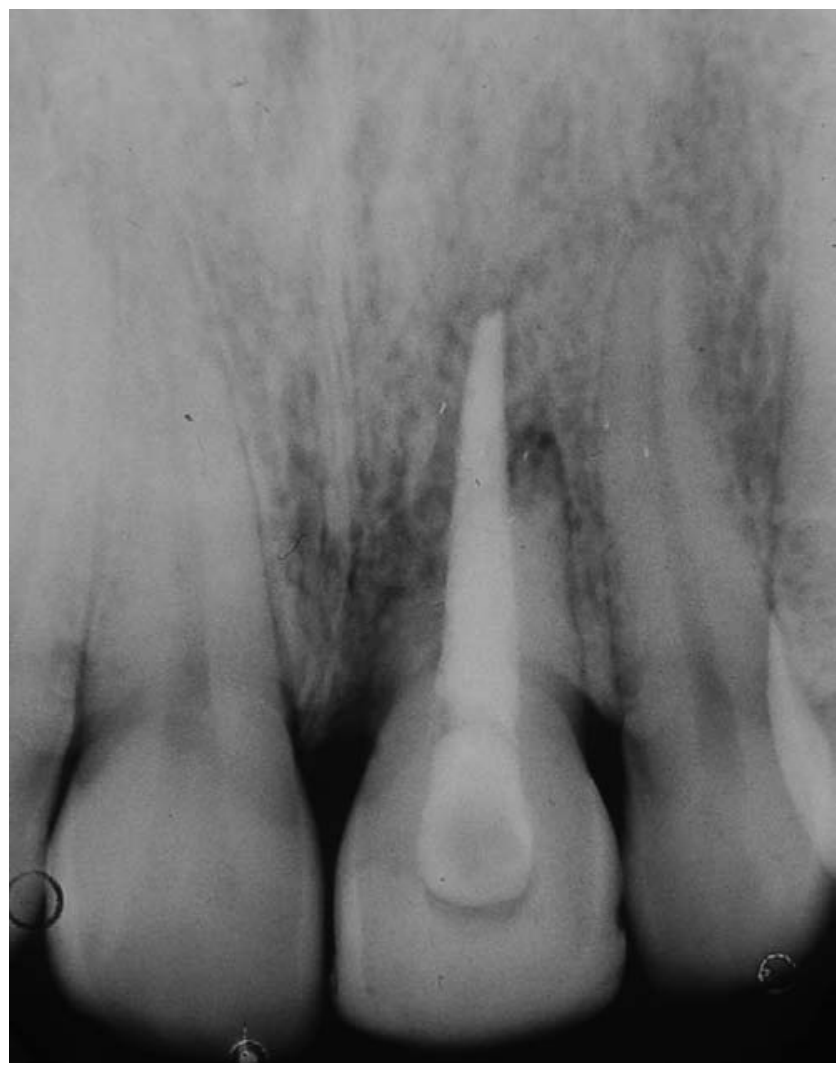

Fig 9. Radiograph of a maxillary left central incisor which had been replanted 13 years earlier. Extensive trauma induced replacement resorption is evident. Although the tooth will ultimately require implant replacement the rate of resorption is slow.
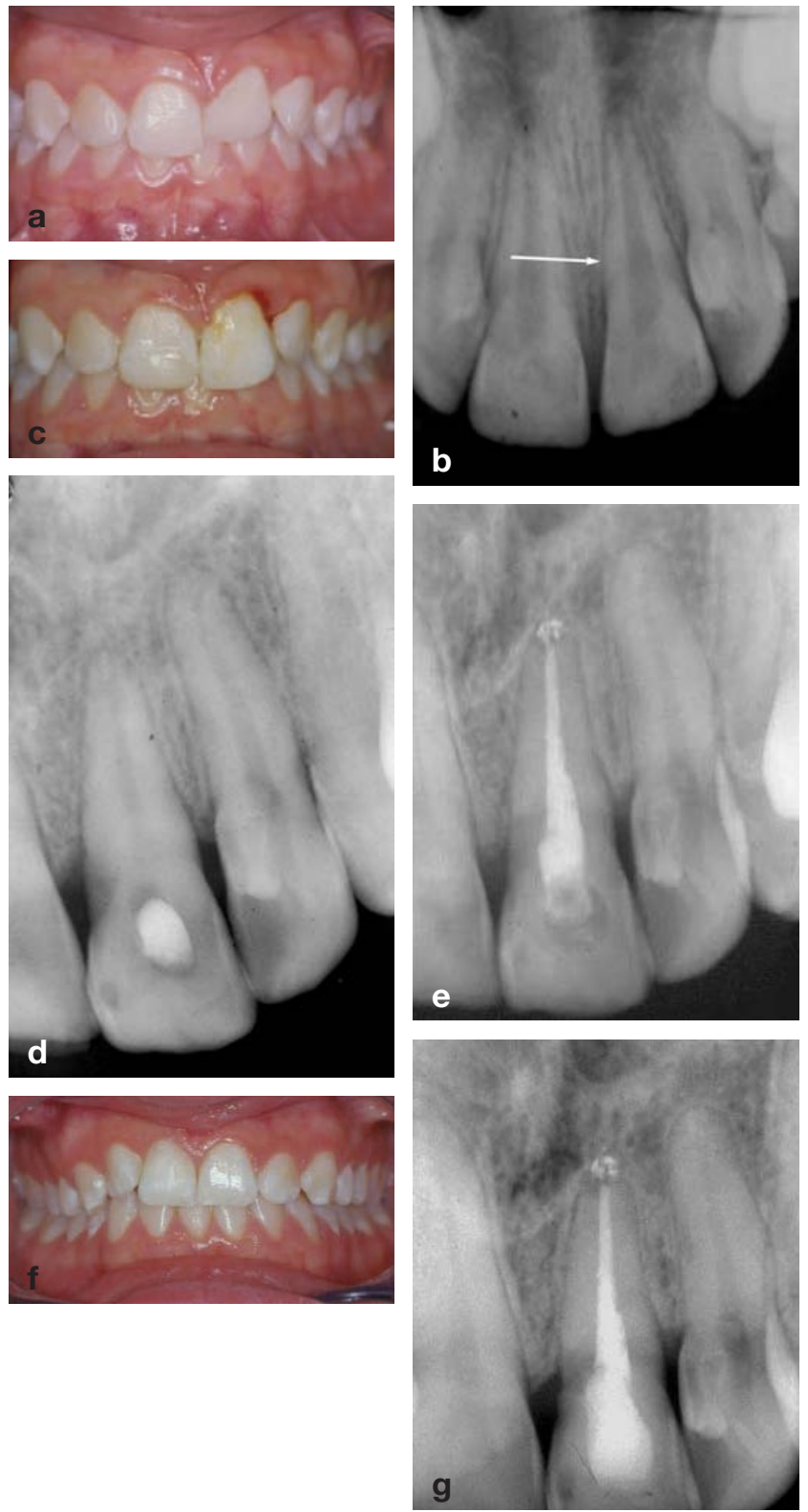

Fig 10a. Labial appearance of an ankylosed maxillary left central incisor in a 13 year old female. The tooth had been intruded at age 8 .

Fig 10b. Radiographically the maxillary left central incisor shows a discernable periodontal ligament image over the majority of the root surface but in one region it appears to be lacking (arrow).

Fig 10c. Labial appearance after intentional replantation into an extruded position. After careful extraction of the tooth, Emdogain was applied to the conditioned root surface prior to replantation.

Fig 10d. Radiograph of repositioned tooth 3 months after treatment which included root canal preparation and the placement of a

$$
\text { Ledermix paste intra-canal dressing. }
$$

Fig 10e. Radiograph taken after root filling 6 months following repositioning and Emdogain application. Slight extruded AH26 is present periapically.

Fig 10f. Eleven-year follow-up shows the tooth had continued normal eruption and the gingival contour is symmetrical.

Fig 10g. A radiograph taken at the 11-year follow-up shows no sign of ongoing resorption. The slight amount of AH26 sealer periapically appears to have been well tolerated.

Ankylosis in the developing dentition can severely disrupt arch formation and some form of early clinical intervention is desirable. In some cases of ankylosis and limited replacement resorption, a surgical repositioning procedure can be attempted to restore arch integrity. 

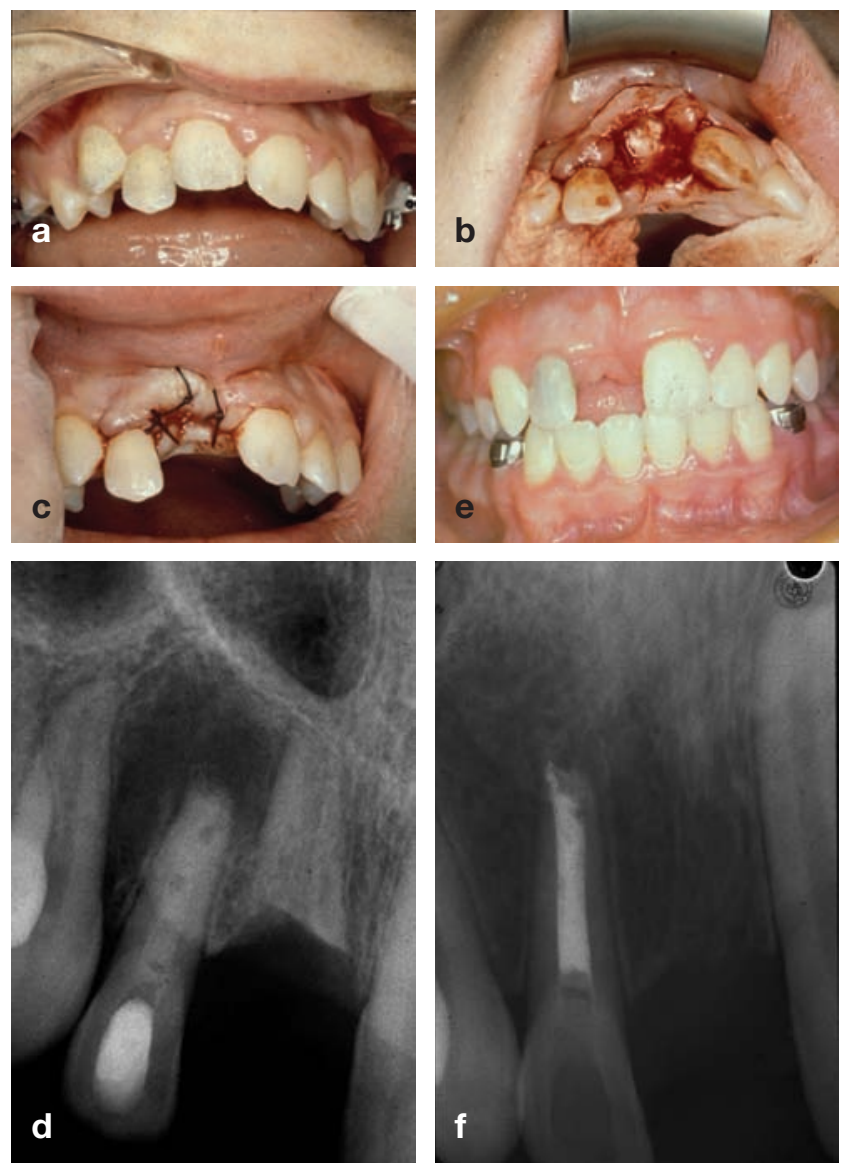

Fig 11a. Labial appearance of an ankylosed maxillary right central incisor in a 14 year old male.

Fig 11b. After flap reflection, the ankylosed tooth was decoronated and submerged, preserving the crown for use as a temporary bonded bridge.

Fig 11c. Labial view after the suturing of the flaps.

Fig 11d. Radiograph showing the maxillary central incisor root following decoronation. (The maxillary right lateral incisor had a

long-term calcium hydroxide dressing to induce apical closure).

Fig 11e. Labial appearance 3 years later showing satisfactory alveolar development.

Fig 11f. Radiograph taken at the same time as Fig 11e shows evidence of alveolar growth and total resorption of the residual root structure.

Courtesy Associate Professor John McNamara.

This procedure can be supplemented with the application of Emdogain (Biora AB Malmo, Sweden) to the affected root area in an attempt to repopulate the denuded surface with cementoblasts. A successful example of this form of management, with a long-term follow-up, is shown in Fig 10. Even if re-ankylosis and replacement resorption continue, the damage to arch development can often be avoided or minimized.

In cases of ankylosis with advanced replacement resorption a decoronation and submergence procedure is recommended. ${ }^{13}$ This allows ongoing alveolar growth both vertically and axially, and it facilitates the uncomplicated transition to implant therapy when appropriate. An example of this approach is shown in Fig 11.

\section{Infection induced dental resorption}

The response of the dento-alveolar apparatus to infection is characterized by inflammation which may result in tooth resorption. This may be a consequence of infective endodontic pathosis alone or superimposed on trauma induced resorption. These infection induced resorptions, which are generally termed inflammatory root resorptions, may occur as internal resorptions, external resorptions or combined internal-external lesions. These infection induced resorptions can vary widely in complexity but will generally respond favourably to therapy aimed primarily at removing the infective agent. Additional impetus to resorption control can be provided by the use of anti-clastic therapeutic agents such as Ledermix Paste (Lederle Pharmaceuticals Wolfratshausen Germany) used as intra-canal medicaments. Other materials such as calcium hydroxide or ProRoot MTA (Dentsply Tulsa Dental, Johnson City, Tennessee, USA) can also be used to stimulate hard tissue formation on resorbed root surfaces. ${ }^{14}$

\section{Internal inflammatory (infective) root resorption}

Internal inflammatory resorptions may be classified according to location as: (1) apical and (2) intraradicular.

\section{(1) Apical}

A recent study has shown that apical internal inflammatory resorption is far more common in teeth with various inflammatory periapical pathoses than previously thought. ${ }^{15}$ The study showed that 74.7 per cent of teeth with periapical lesions had varying degrees of apical internal resorption, ranging from slight (grade 1) to severe (grade 4). Radiographically, apical internal resorption may be difficult to diagnose when the resorptions are of the lower grades as described by Vier and Figueiredo, ${ }^{15}$ but should be discernable in grades 3 and 4 lesions. This study has important clinical implications in root canal preparation. There are two approaches to the endodontic management of apical internal resorption. The first is to extend instrumentation only to the position of the resorption with the expectation that with the removal of micro-organisms followed by root canal filling, hard tissue repair will occur in the resorbed apical region of the tooth. The second approach is to enlarge and prepare the apical region, either with hand or rotary filing techniques, to include the resorbed region and then root fill to the root canal "terminus". ${ }^{16}$ At this stage an evidenced based, randomized prospective study to compare the longterm results of the two approaches has not been carried out. Until such evidence becomes available, treatment to the position of the resorption is recommended as a clinical means of achieving biological repair of the resorbed apex.

In some instances, gross resorption can result from endodontic pathosis as shown in Fig 12a and treatment can provide considerable clinical challenges which must be carefully evaluated by a practitioner who may consider referral to an endodontist. Treatment involves root canal preparation to the position of the resorption followed by long-term calcium hydroxide intra-canal 

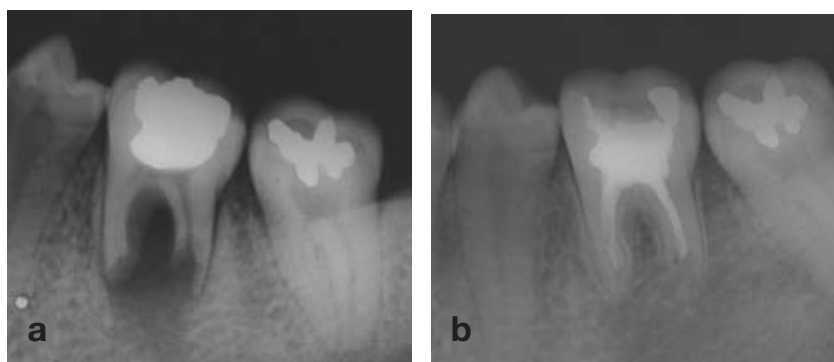

Fig 12a. Radiograph showing infection induced apical internal root resorption and associated periradicular infection.

Fig 12b. Radiograph taken 2 years later showing periradicular repair and control of the root resorption.

medication, and a sound overlying and double coronal seal of Cavit and GIC. Cuspal protection during longterm medication is also very important. Once periapical and resorption repair has occurred, the canals can be filled with gutta-percha and a sealer. An example of the healing of an extreme example of apical internal resorption is shown in Fig 12b. ProRoot MTA could also be used as there is increasing evidence for its efficacy when used in similar resorptions. ${ }^{17}$

\section{(2) Intraradicular}

Internal resorption fully contained within an otherwise intact root will be referred to as intraradicular internal inflammatory resorption. Infection induced internal resorption can be recognized as round or ovalshaped radiolucencies contained within the tooth root and examples are shown in Figs 13a, 13b and 14a.

A common finding is a large accessory canal communicating from the periodontal ligament to the resorbed area; this may have allowed the passage of a collateral blood supply which probably played an important role in the development and maintainance of the internal resorptive process. Treatment generally
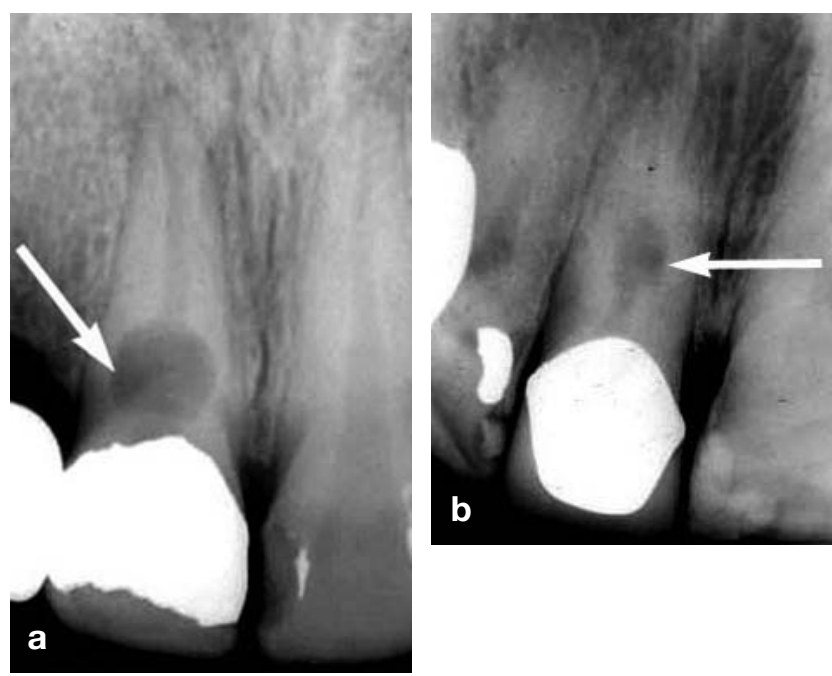

Fig 13a. Radiograph of a maxillary right central incisor showing infection induced internal radicular inflammatory resorption. Arrow indicates the likely presence of a large interconnecting accessory canal.

Fig 13b. Radiograph of a maxillary right central incisor showing an infection induced radicular internal inflammatory resorption. Arrow indicates the likely presence of an accessory canal.
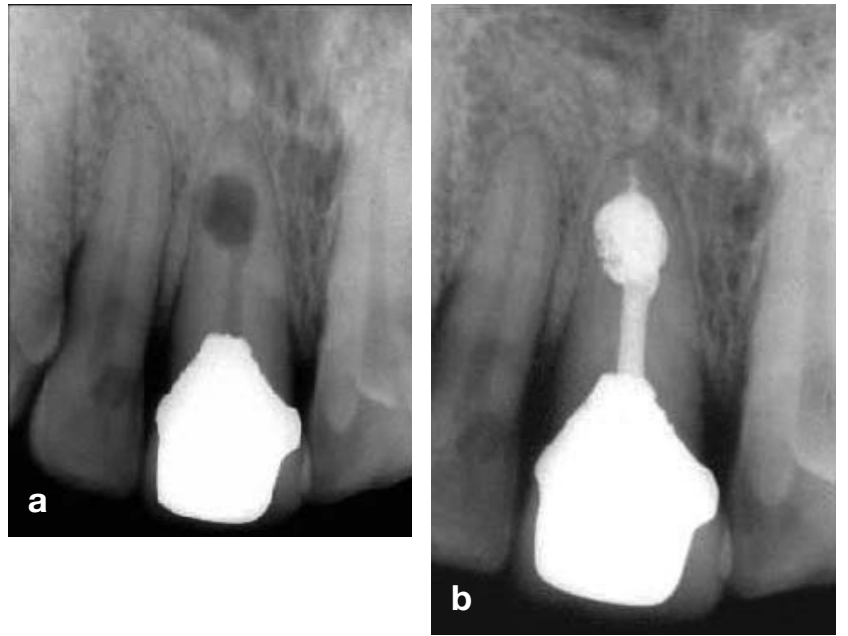

Fig 14a. A further example of infection induced radicular inflammatory root resorption which was treated endodontically including the use of sonication.

Fig 14b. Radiograph taken after obturation of the root canal with gutta percha and AH26 using a vertically condensed heated gutta percha technique.

consists of the preparation of the canal to the apical foramen with particular emphasis on irrigation and ultrasonication so that the resorbed area is cleansed as thoroughly as feasible. The obturation of the canal can be achieved by a variety of techniques including hot vertically condensed gutta-percha, Obtura-delivered hot gutta-perch $\mathrm{a}^{18}$ and more recent innovations such as the Microseal technique.

An example of a hot vertically condensed root filling of an intraradicular internal resorption is shown in Fig 14b.

\section{External inflammatory root resorption}

Classically, this type of external root resorption occurs when infection is superimposed on a traumatic injury - usually following replantation of an avulsed tooth or a luxation injury. Nevertheless it can also be induced in some cases of endodontic pathosis as shown in Fig 15a. Despite the degree of external inflammatory resorption, treatment can be successful (Fig 15b).

A prerequisite for external inflammatory root resorption is damage to the normally protective
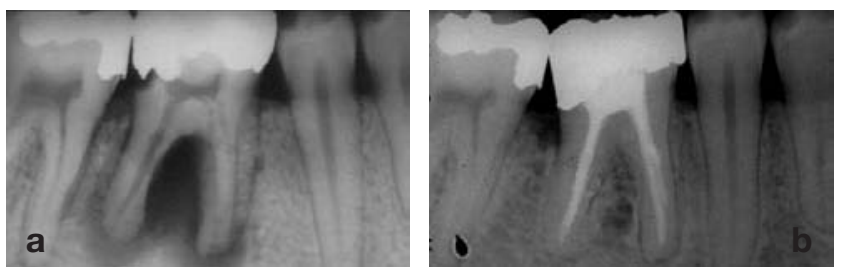

Fig 15a. A mandibular first molar with radiographic evidence of extensive infection induced external inflammatory root resorption and an associated periradicular radiolucency indicative of an extensive inflammatory response to endodontic pathosis. Fig 15b. Radiograph taken 1 year after endodontic therapy which consisted of the removal of the coronal restoration, preparation of the root canal system with endodontic files, liberal irrigation and sonication with EDTAC, $1 \%$ sodium hypochlorite, and EDTAC used sequentially, long-term intra-canal dressings of calcium hydroxide and finally obturation with gutta percha and AH26. 

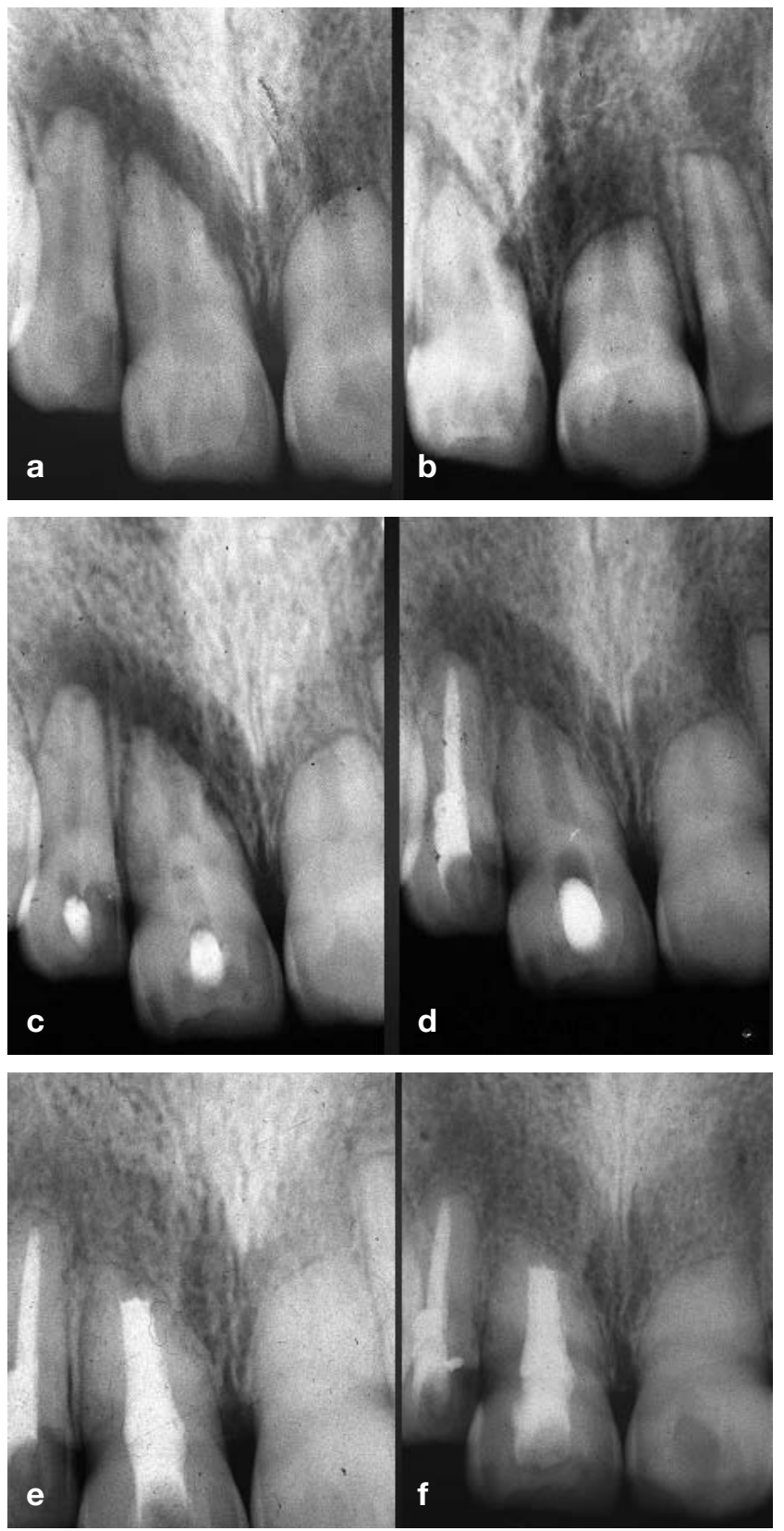

Figs $16 \mathrm{a}$ and $16 \mathrm{~b}$. Radiographs of maxillary central incisors of a 14 year old female replanted within 5 minutes of their avulsion reveal signs of infection induced external inflammatory root resorption in the right central incisor (arrows) and a periapical radiolucency associated with the right lateral incisor. The left central incisor remains free of signs of pathosis.

Fig 16c. Radiographic appearance 3 months after the commencement of endodontic therapy in the maxillary right central and

lateral incisor teeth. Calcium hydroxide was used as the intra-canal medicament.

Fig 16d. Radiograph after a further 3 months dressing with calcium hydroxide, shows evidence of repair of the resorbed root surface and apical dome formation. Good periapical repair is also evident in the root filled lateral incisor.

Fig 16e. Radiograph taken at the time of root filling the maxillary central incisor 12 months after the injury. Note the almost complete calcification of the maxillary left central incisor which provides positive evidence of successful revacularization.

Fig 16f. A 20-year follow-up radiograph shows no evidence of any further resorption.

cementum/cementoid which then initiates surface resorption exposing the underlying dentine to the passage of bacteria or their metabolites from the root canal to the external root surface. A normal inflammatory response ensues including the activation of clastic cells which results in resorption of both tooth and bone. As the inflammatory response is chronic in nature it is generally asymptomatic unless the infection becomes acute, in which case the tooth will become tender to touch and there may be the development of an overlying swelling.

Radiographically external inflammatory root resorption can be recognized by bowl-like radiolucencies in both the tooth root and the adjacent bone. This is a progressive form of root resorption which will ultimately result in tooth loss. However, in most instances external inflammatory root resorption can be stabilized with treatment. As with other forms of infection induced resorption, treatment involves the thorough debridement and preparation of the root canal system. Irrigation is a most important component of this debridement process and the sequential use of $17 \%$ EDTAC, $1 \%$ sodium hypochlorite and a final rinse with EDTAC solution has been shown to be a most effective regimen resulting in a dentine surface devoid of smear layer. ${ }^{19}$ Such dentine preparation facilitates the diffusion of medicaments such as Ledermix paste through dentine to the external root surface where the corticosteroid and antibiotic components of the paste can exert a positive effect on the clastic cells responsible for the resorptive processes. ${ }^{20,21}$

Calcium hydroxide has been widely used in the treatment of external inflammatory root resorption ${ }^{22}$ as illustrated in a case of external inflammatory root resorption shown in Fig 16 with a 20-year follow-up.

An alternative approach involves the use of Ledermix paste as the initial intra-canal medicament to act as an anti-clastic agent. This root canal medication is replaced at six-weekly intervals for a period of approximately three months and then if there has been radiographic signs of resorption control, calcium hydroxide can be used to influence hard tissue deposition on resorbed root surfaces. This sequential use is shown in Figs $17 \mathrm{a}-17 \mathrm{~d}$ which involved a case of external inflammatory resorption observed six months following the replantation of an incompletely developed central incisor. A 10-year follow-up radiograph is shown in Fig 17e.

Prevention of inflammatory root resorption: A tooth with complete root development which has been subjected to avulsion, intrusion or a severe luxation injury should, after replantation or repositioning, have the pulp removed as soon as possible and the canal dressed with Ledermix paste so that its anti-clastic effect can be exerted in the early phases of the healing process. ${ }^{23}$ If calcium hydroxide is to be used as the initial dressing material, treatment should be delayed for at least two weeks to avoid cellular necrosis and ankylosis. ${ }^{24-26}$ In a tooth with an immature apex, with a diameter greater than $2 \mathrm{~mm}$, there is a chance of revascularization in all the above injuries, provided in the case of a replanted tooth that the extra-oral period 

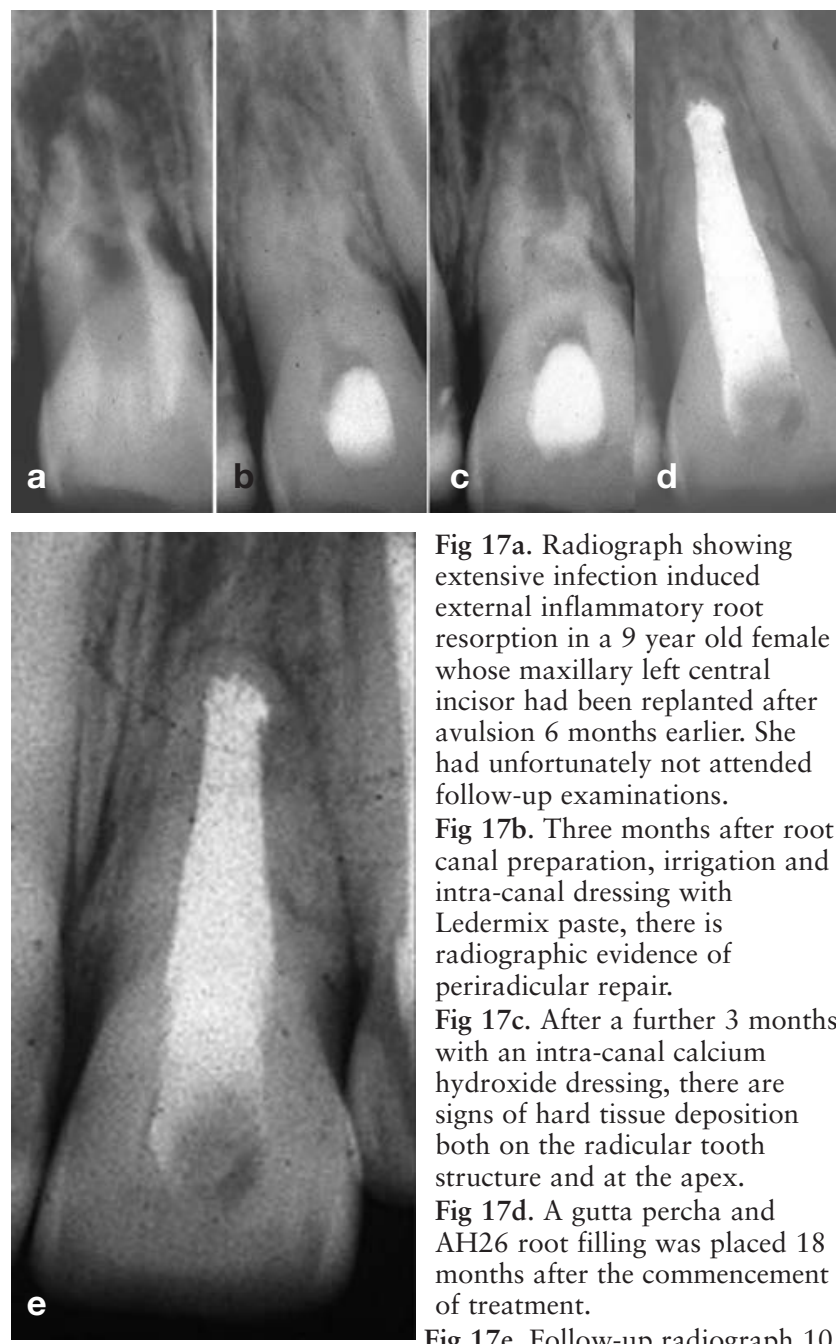

Fig 17a. Radiograph showing extensive infection induced external inflammatory root resorption in a 9 year old female whose maxillary left central incisor had been replanted after avulsion 6 months earlier. She had unfortunately not attended follow-up examinations.

Fig 17b. Three months after root canal preparation, irrigation and intra-canal dressing with Ledermix paste, there is radiographic evidence of periradicular repair.

Fig $17 \mathrm{c}$. After a further 3 months with an intra-canal calcium hydroxide dressing, there are signs of hard tissue deposition both on the radicular tooth structure and at the apex.

Fig 17 d. A gutta percha and AH26 root filling was placed 18 months after the commencement of treatment.

Fig 17e. Follow-up radiograph 10 years after root filling with gutta percha and $\mathrm{AH} 26$, shows periradicular repair and no sign of further resorption.

is relatively short (30 minutes) or the tooth has been stored in a medium which maintains the viability of the periodontal ligament. Teeth with wide apices subjected to such trauma should be monitored carefully at monthly intervals for three months and at longer intervals thereafter. If radiographic signs of inflammatory root resorption become evident, immediate endodontic intervention is required.

\section{Communicating internal-external inflammatory resorption}

Where resorption has extended from an internal inflammatory resorption to involve the external surface a communicating lesion is created. This can be recognized radiographically by a radiolucency within the tooth structure extending to the exterior surface and the surrounding bone. While treatment is possible, it is complex and referral to an endodontist is suggested or implant therapy could be considered.

Endodontic treatment is carried out in the coronal segment to the level of the resorptive defect where various materials and medicaments can be used to induce calcification at or within the resorptive lesion. Traditionally, calcium hydroxide has been used for this
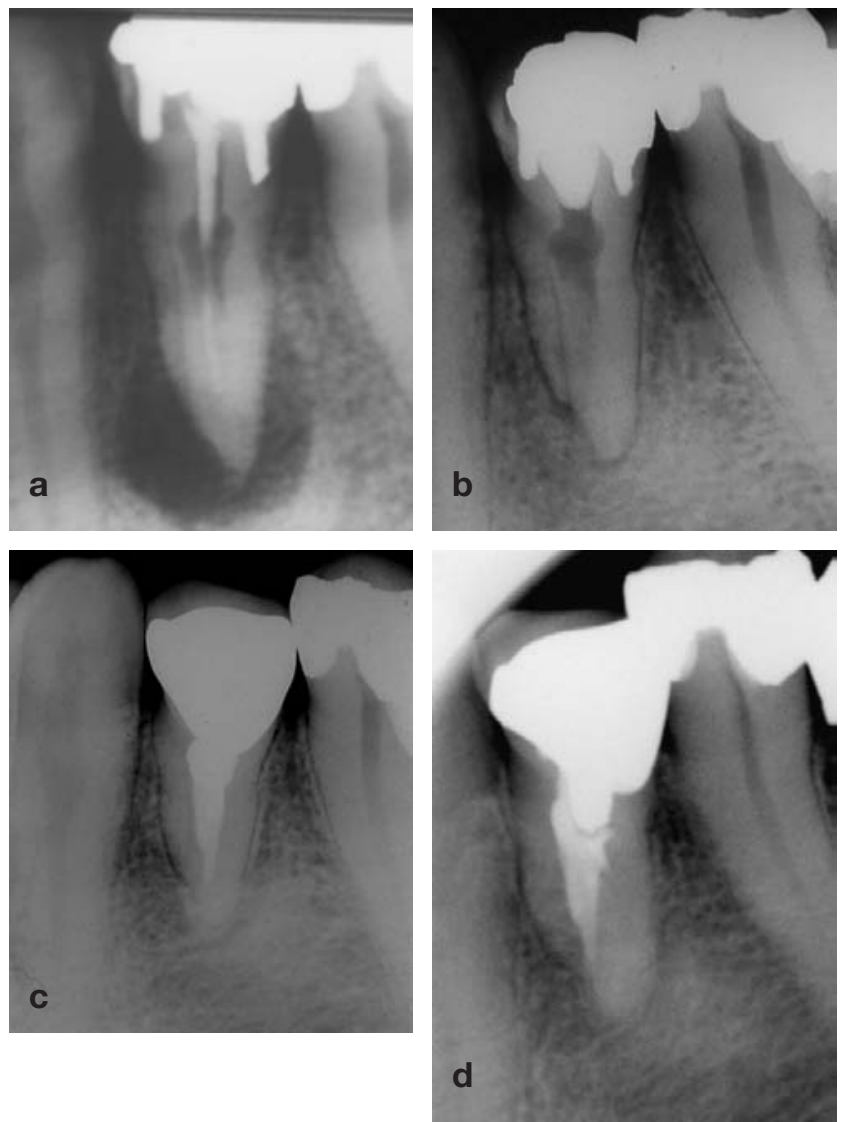

Fig 18a. Mandibular left first premolar of a 30 year old male showing an infection induced communicating internal-external inflammatory root resorption and a related periradicular inflammatory lesion.

Fig 18b. Radiograph showing evidence of good healing following the preparation of the root canal system and long-term dressing with a calcium hydroxide paste.

Fig 18c. Radiograph following filling of the root canal system with gutta percha and $\mathrm{AH} 26$ and the placement of a crown.

Fig 18d. A 35-year follow-up radiograph shows continuing periradicular health and no evidence of further resorption.

purpose and an example where this has been employed is shown in Fig 18 with a 35-year follow-up.

Another approach involves the topical application of $90 \%$ aqueous trichloracetic acid to the resorptive tissue following endodontic preparation to the level of the resorptive defect. Trichloracetic acid is applied for 1-2 minutes on a mini-applicator or a small cotton pellet attached to an endodontic file. This will induce a sterile coagulation necrosis of the resorptive tissue which can act as a nidus for calcification - a process labelled by the author as "scaffolding". An example of such a treatment of an extensive communicating lesion is shown in Fig 19 with a 13-year follow-up period.

\section{Hyperplastic invasive resorptions}

The third group of dental resorptions are insidious in nature and generally present complex therapeutic challenges. In these cases, resorbing tissue invades the hard tissues of the tooth in a destructive, and apparently uncontrolled fashion, akin to the nature of some fibroosseous lesions such as fibrous dysplasia. ${ }^{27}$ An important distinguishing factor for this third group of 

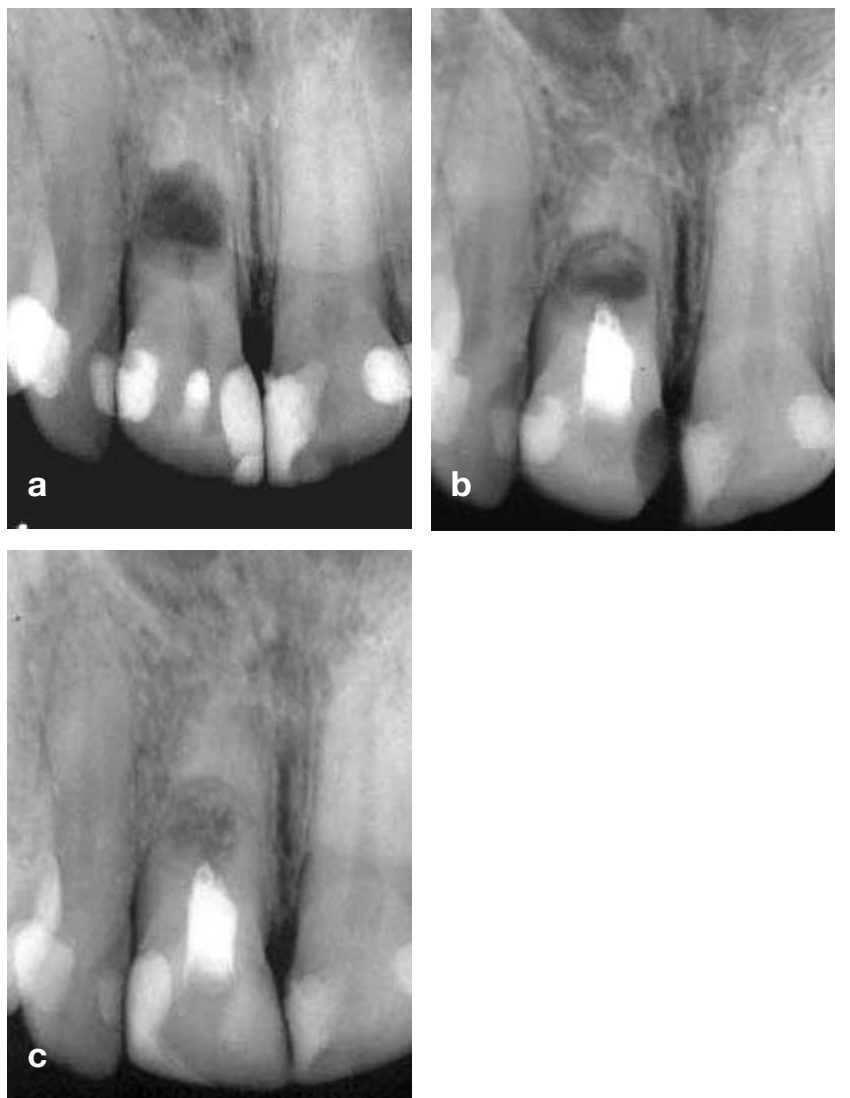

Fig 19a. Radiograph of a maxillary right central incisor of a 39 year old female showing an extensive infection induced communicating internal-external inflammatory root resorption.

Fig 19b. Radiograph taken at the time of filling the root canal with gutta percha and AH26, 5 months after treatment of the resorptive lesion by the topical application of trichloracetic acid followed by dressing with calcium hydroxide. There is radiographic evidence of hard tissue deposits within the resorptive defect.

Fig 19c. Radiograph taken 13 years after "scaffolding" therapy showing extensive hard tissue deposition within the resorptive lesion.

resorptions is that, unlike the first two types of resorption, simple elimination of the cause of the lesion is ineffective in arresting their progress. Total removal or inactivation of the resorptive tissue is essential if recurrence (or concurrence) is to be avoided. Concurrence indicates the incomplete removal of the resorptive tissue at the time of treatment and recurrence is the re-establishment of the resorptive process. The reason for recurrence or concurrence is probably due to the invasive nature of the resorptive tissue whereby small infiltrative channels are created within the dentine and these may interconnect with the periodontal ligament in positions more apical to the main resorptive defect. ${ }^{28}$ Unless the tissue in these infiltrative channels is inactivated the resorptive process will continue. While these resorptions are not considered to be neoplastic, their aggressive characteristics can seem to be similar. At the present time one proven therapy for inactivation is chemical in nature but surgical and other technological modalities may also be applied.

These hyperplastic invasive resorptions may have a pulpal (internal) or a periodontal origin (external).
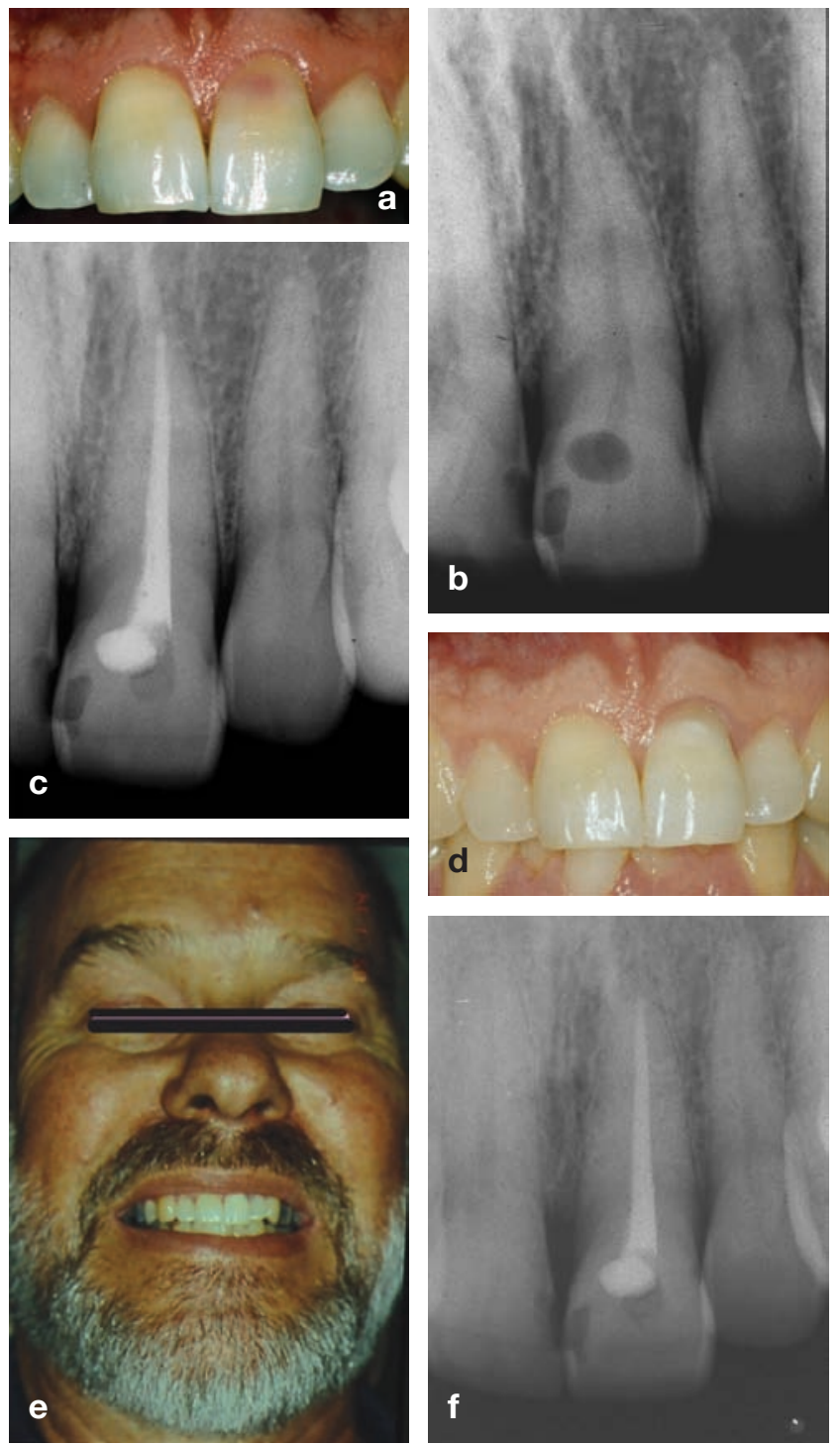

Fig 20a. A rare example of hyperplastic internal replacement (invasive) resorption is shown in the maxillary left central incisor of a 38 year old male who had received a blow to the tooth 2 months earlier. Pink discolouration is evident, confined to the crown of the affected tooth. The location of this pink discolouration should be contrasted with a later example of invasive cervical resorption shown in Fig 24a.

Fig 20b. Radiograph showing a coronal radiolucency. A diagnosis of hyperplastic internal replacement (invasive) resorption is based on the history of recent trauma and the clinical and radiographic appearance of this tooth.

Fig 20c. Radiograph following pulpectomy and root filling with gutta percha and $\mathrm{AH} 26$.

Fig 20d. Labial view following treatment.

Fig 20e. Follow-up examination 10 years later shows a satisfactory aesthetic result.

Fig 20f. Radiograph at the 10-year follow-up examination shows no sign of periradicular pathosis or ongoing resorption.

Critical to the clinical use of therapies aimed at controlling internal and external invasive tooth resorptions is the correct diagnosis of the type and localization of the resorption.

Hyperplastic resorptions may be subdivided into internal replacement (invasive) resorption, invasive coronal resorption, invasive cervical resorption and invasive radicular resorption.

Australian Dental Journal Endodontic Supplement 2007;52:1. 

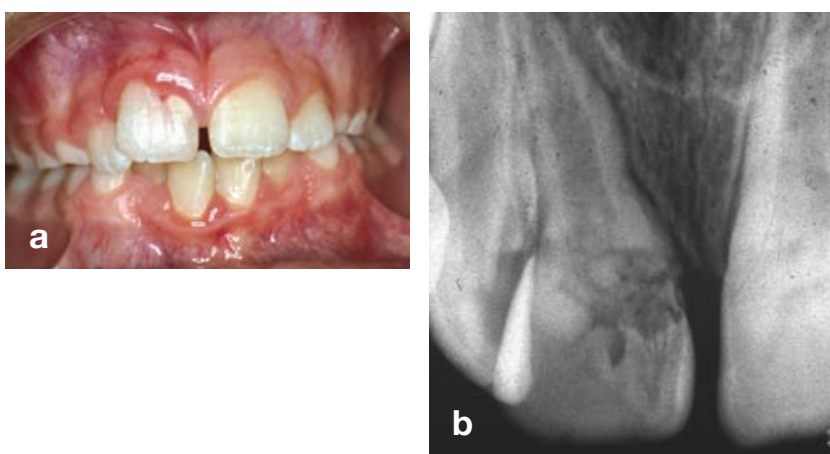

Fig 21a. Hyperplastic invasive coronal resorption in the maxillary right central incisor of an 11 year old male, the resorption being apparently associated with an area of hypomineralization of the crown.

Fig 21b. Radiograph of the maxillary right central incisor showing an extensive irregular radiolucency indicative of hyperplastic invasive coronal resorption.

\section{Internal replacement (invasive) resorption}

This type of resorption is relatively rare $^{29}$ and may appear clinically as a pink area in the crown of the affected tooth as shown in Fig 20a. However, the clinician should recognize that a pink appearance in the crown of a tooth is indicative of highly vascular resorbing tissue which has removed sufficient dentine and enamel to allow it to be visible through the thin overlying tooth substance. While this may be internal in origin, particularly if there has been a history of recent trauma, it more commonly arises from an external periodontal source. The location of the pink spot is more likely to be entirely within the crown of the tooth in internal replacement (invasive) resorption.

Radiographically it may appear as an irregular outline within the image of the root canal or as an extension of it as shown in the oval radiolucency in Fig 20b. Management consisting of pulpectomy, curettage of the resorptive defect and root filling will generally control the resorptive process as illustrated in Figs 20c-Fig 20d. The long-term follow-up examination of that treatment is shown in Figs 20e-20f. In more extensive cases, the resorptive tissue may communicate with the periodontal ligament and pulpectomy should be supplemented by the careful topical application to the defect of $90 \%$ aqueous trichloracetic acid on a small cotton wool pellet (size 00) or a mini-applicator. This will aid in the inactivation of any communicating resorptive tissue. Generally, this treatment can be followed by the insertion of a conventional root filling although in communicating lesions there may be occasions where mineral trioxide aggregate (ProRoot MTA) may be used to advantage in sealing the defect prior to the placement of a root filling.

\section{Invasive coronal resorption}

This rare condition generally develops in erupting teeth where a localized coronal enamel defect allows the invasion of aggressive hyperplastic resorptive tissue, as illustrated in Fig 21a. In this case a pink resorptive defect can be observed in an area of hypomineralization on the labial surface of the crown of the erupting central incisor tooth. Invasive coronal resorption has also been observed in teeth which have been injured by the intrusion of a primary tooth.

Radiographically the image of the resorptive defect is generally irregular in outline and, depending on its extent, the radiolucency may extend both coronally and into the radicular tooth structure as illustrated in Fig 21b.

Management: Treatment is directed towards the total removal or inactivation of all resorptive tissue and the restoration of the coronal defect. This may be achieved by physical curettage of the defect with round burs and hand instruments, but is more conveniently and effectively treated by the topical application of $90 \%$ aqueous trichloracetic acid, curettage, endodontic therapy if there is pulp involvement, and restoration of the defect with a glass ionomer cement. Orthodontic extrusion to render the defect into a supragingival position may supplement treatment if the resorption extends deeply as illustrated in Fig 21b. While the pulp can be completely or partially retained in some cases, pulpectomy and root canal filling will be required in the more extensive resorptions.

\section{Invasive cervical resorption}

Characterized by its cervical location and invasive nature, this insidious resorptive process may occur in any tooth in the permanent dentition. ${ }^{30}$ In the absence of treatment, invasive cervical resorption leads to progressive and usually destructive replacement of tooth structure. Resorption of coronal dentine and enamel often creates a clinically obvious pinkish colour in the tooth crown as highly vascular resorptive tissue becomes visible through thin residual enamel. In other instances there may be no obvious outward sign of this process and its detection may be by routine radiographs. The condition is usually painless unless there is superimposed secondary infection when pulpal or periodontal symptoms may arise. Invasive cervical resorption has been and continues to be, misdiagnosed as a form of internal resorption. This misunderstanding could possibly be attributed to the early descriptions of internal resorption by Gaskill ${ }^{31}$ in 1894 and by Mummery ${ }^{32}$ in 1920, which included teeth showing "pink spots".

Although the cause of this condition remains obscure, potential predisposing factors have been identified $^{11}$ and these include trauma, orthodontic treatment and intra-coronal bleaching as sole factors or in combination.

The clinical management of invasive cervical resorption varies with the degree of progression of this complex resorptive process but the basic aim remains the same, namely the total removal or inactivation of resorptive tissue and the restoration or reconstitution of the resorptive defect.

A clinical classification has been developed as a guideline for treatment planning and for comparative 


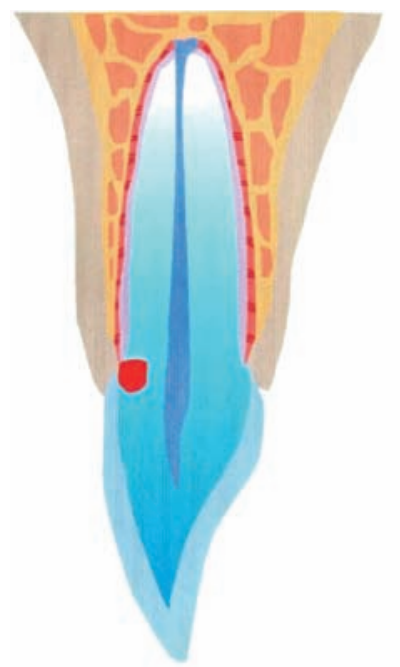

Class 1

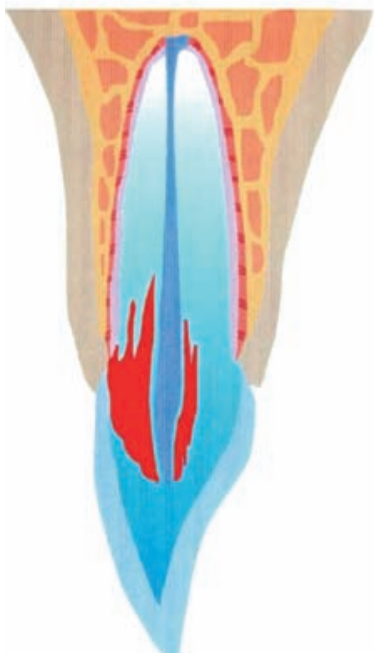

\section{Class 3}

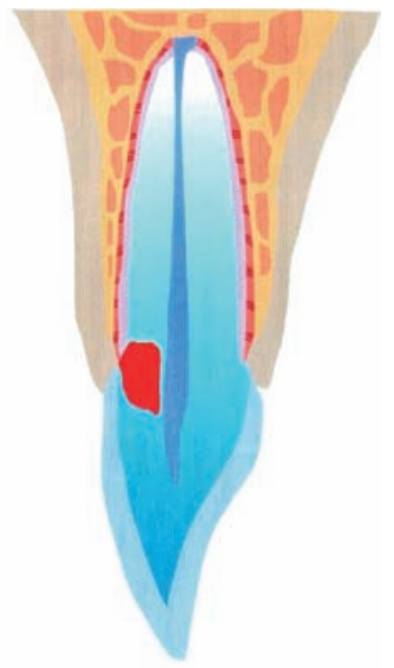

Class 2

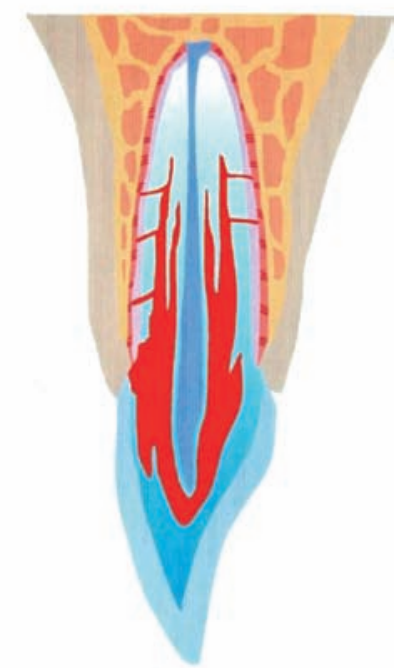

Class 4
Fig 22. Clinical classification of invasive cervical resorption. Reproduced from Heithersay GS, Quintessence Int 1999;30:83-95, courtesy Quintessence International.

clinical research. ${ }^{11}$ The classification is shown diagrammatically in Fig 22. If an invasive cervical resorption has been diagnosed at a Class 1 or Class 2 stage, the resorptive tissue is fibro-vascular in character and the pulp is walled off by a protective pre-dentine and dentine barrier (Fig 23).

Traditional methods of treatment consist of curetting the active tissue from the resorption cavity and restoring the defect with a suitable restorative material. An alternative method, which utilizes the topical application of $90 \%$ aqueous trichloracetic acid, curettage and restoration, has been outlined and clinically assessed. ${ }^{30}$ One advantage of this approach is haemorrhage control which can prove a problem with conventional treatment. As the effect of trichloroacetic acid is to cause coagulation necrosis, the resorptive tissue is rendered avascular. In addition there is a tendency for recurrence of this type of resorption and

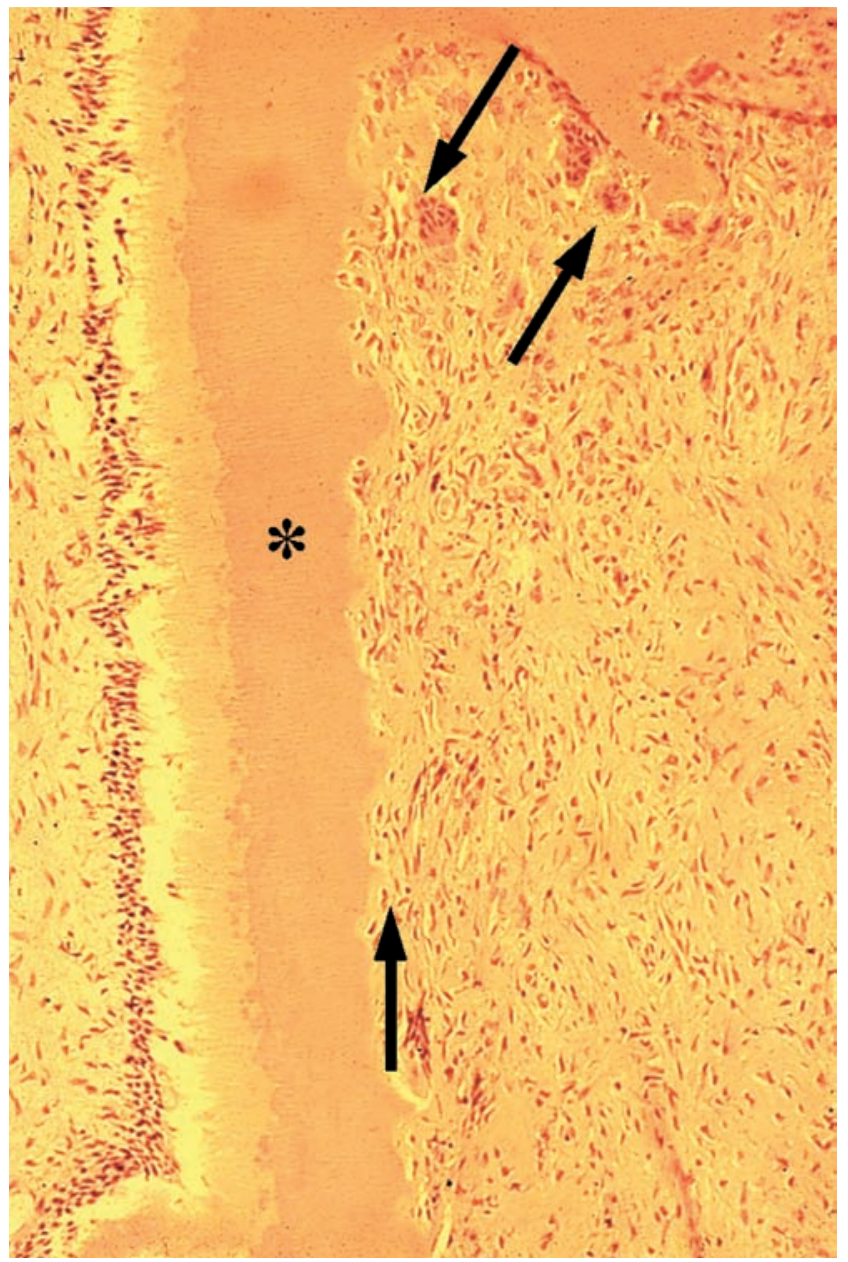

Fig 23. Histologic appearance of an incisor tooth with Class 2 invasive resorption. An intact layer of dentine and predentine on the pulpal aspect $\left({ }^{*}\right)$ separates the pulp from the resorbing tissue. The resorption cavity is filled with a mass of fibrovascular tissue with active mononucleated and multi-nucleated classic cells lining resportion lacunae (arrows). (Haematoxylin-eosin stain; original magnification x 40.) Courtesy Assoc Prof J McNamara.

Reproduced from Heithersay GS, Quintessence Int 1999;30:27-37, courtesy Quintessence International.

the inactivation of adjacent and potentially resorptive cells by trichloroacetic acid is a further reason for its utilization.

The following case report illustrates this treatment regimen applied to a Class 2 category invasive cervical resorption which occurred in a maxillary right central incisor of a 21 year old female who had a history of dental trauma to the tooth at age 12 . The clinical and radiographic appearance is shown in Figs 24a-24b. Rubber dam had been applied using a cuff technique after the protective application of glycerol to adjacent soft tissues and the placement of a glycerol impregnated cotton roll into the labial sulcus (Fig 24c).

A small cotton pellet (size 00 divided in half) which had been dipped into a very small quantity of a $90 \%$ aqueous solution of trichloroacetic acid and then dampened on gauze, was applied for 1-2 minutes with gentle pressure to the resorptive lesion which was accessible through an enamel defect near the gingival margin (Fig 24d). The pressure was slowly increased as 

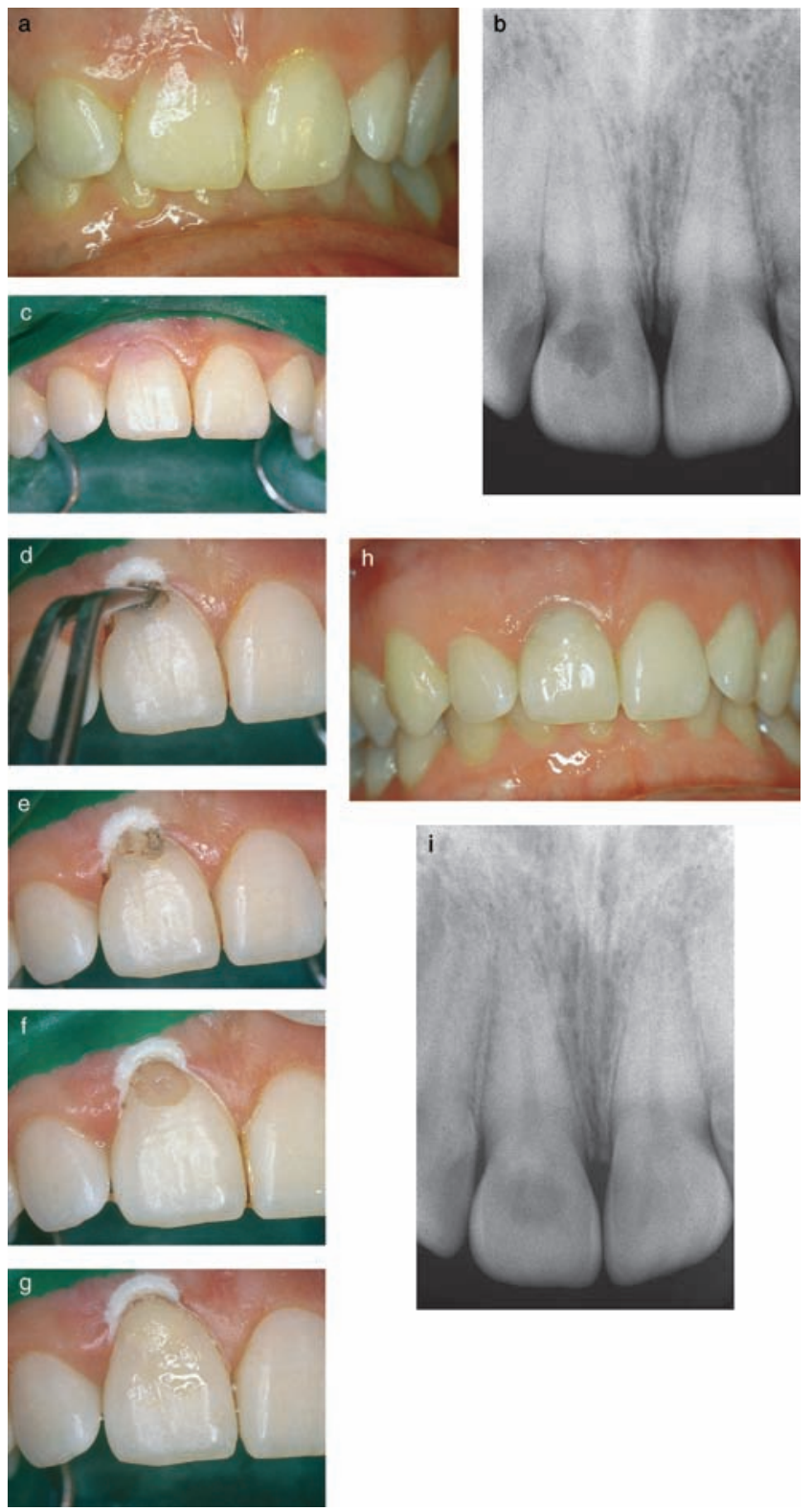

the medicament caused progressive coagulation necrosis of the resorptive tissue and there was collapse of the thin overlying enamel (Fig 24e). The devitalized avascular tissue was curetted from the resorption cavity, which was then carefully checked under magnification with an enhanced light source. This examination revealed an intact smooth dentine floor cavity with no communication with the dental pulp (Fig 24f). The cavity margins were then smoothed with a high speed tungsten carbide bur under water spray and the defect was restored with a glass-ionomer cement, protected with a light activated unfilled bonding resin (Fig 24g). Follow-up examinations to five years did not reveal any evidence of pulpal or periapical pathosis or continuation of the resorptive process, and the restoration and adjacent gingival tissues were assessed as most satisfactory (Figs 24h-24i).

If on removal of the resorptive tissue, there is obvious pulp involvement, pulpectomy should be carried out accessing the canal via the resorption cavity to retain as much residual tooth structure as possible.
Fig 24a. The maxillary right central incisor of a 21 year old woman shows a pink discolouration on the labial aspect of the crown. The tooth had been traumatized 9 years earlier.

Fig 24b. A radiograph of the maxillary right central incisor reveals an irregular radiolucency overlying the root canal with no obvious

extensions into the root canal. This invasive cervical resorptive lesion is classified as Class 2.

Fig 24c. After a protective application of glycerol to adjacent soft tissue, a rubber dam "cuff" has been placed for protection and isolation. This has been supplemented with a glycerol impregnated cotton roll placed in the labial sulcus.

Fig 24d. Trichloracetic acid on a small cotton pellet is applied to the resorptive defect with slowly increasing pressure, so that the resorptive tissue within the cavity undergoes coagulation necrosis. Fig 24e. The appearance of the tissue within the resorptive defect following the application of trichloracetic acid indicates tissue necrosis. The adjacent whitened gingival tissues indicate a limited zone of coagulation necrosis.

Fig 24f. Following curettage of the avascular tissue from the resorption cavity, the glistening dentinal base of the cavity is revealed. The incisal margin of the cavity has been smoothed with high-speed bur under water spray.

Fig 24g. A glass-ionomer restoration has been placed in the cavity, and its surface has been protected with a light-activated unfilled bonded resin.

Fig 24h. Clinical appearance of the tooth 5 years postoperatively.

The original glass-ionomer cement has been faced with a resin composite restoration.

Fig 24i. A 5 year follow-up radiograph of the maxillary right central incisor shows no evidence of periapical pathosis or extension of the treated resorptive lesion.

Reproduced from Heithersay GS, Quintessence Int 1999;30:96-110, courtesy Quintessence International.

When invasive cervical resorptions are diagnosed at the Class 3 and Class 4 stage of development, treatment poses far greater challenges due to the infiltrative and fibro-osseous characteristics of the resorptive lesion. Ectopic bone-like deposits can be observed both within the lesion and at the interface with resorbed dentine as shown in Figs 25a and 25b. In addition, the resorptive tissue creates a series of channels that encircle the root canal and infiltrate into the radicular dentine. A further complicating feature is the interconnection of these infiltrating channels with the periodontal ligament at other locations on the root surface. These complexities must be considered in the management of Class 3 lesions if treatment is to be successful. An illustrative treatment of a Class 3 invasive cervical resorption is shown in the following case report of a maxillary right central incisor of a 19 year old male who had received orthodontic treatment six years earlier. The clinical presentation of the resorption is shown in Figs 26a and $26 \mathrm{~b}$ and the corresponding radiograph is shown in Fig 26c. Following the preparation and protection procedures outlined for the illustrative Class 2 case. Trichloroacetic acid was applied on a small cotton pellet to the resorptive tissue on the palatal aspect of the tooth for approximately three or four minutes (Fig 27a). The medicament was replenished on at least two occasions, and the pressure on the cotton pellet was slowly increased as the tissue within the resorption cavity became progressively avascular due to a process of coagulation necrosis (Fig 27b). In this way the majority of the coronal component of the resorption cavity could be accessed and then simply removed by curettage (Fig 27c). Although an apparent base was 


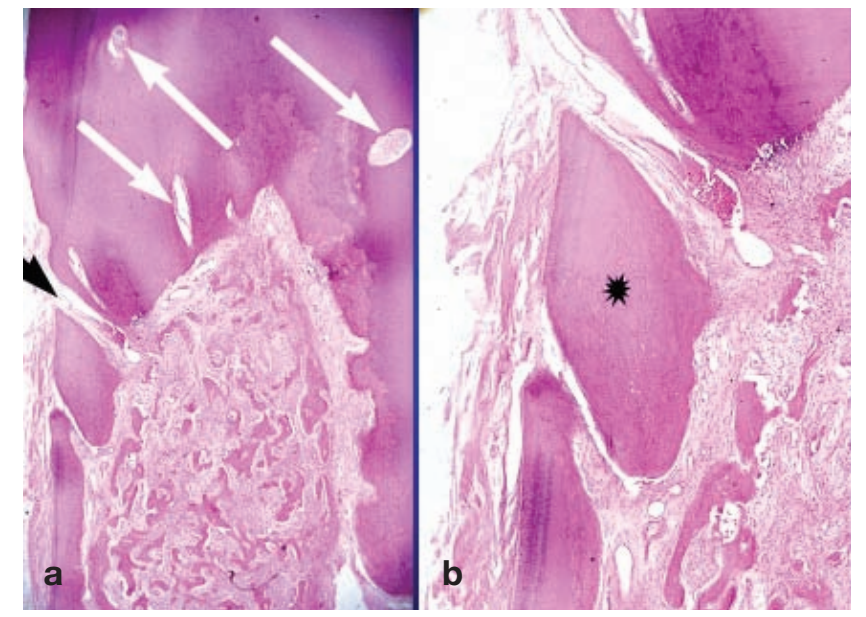

Fig 25a. Histologic appearance of an extensive invasive cervical resorption with radicular extensions. Masses of ectopic calcific tissues are evident both within the fibrovascular tissue occupying the resorption cavity and on resorbed dentine surfaces. In addition communicating channels can be seen connecting with the periodontal ligament (black arrow). Other channels can be seen within the radicular dentine (white arrows). (Haematoxylin-eosin stain; original magnification $\mathrm{x} 30$.)

Fig 25b. Higher magnification of Fig 25a showing communicating channels from the periodontal ligament to the resorbing tissue. An island of hard tissue remains (*), consisting of an external surface of cementum and cementoid, plus some residual dentine but the bulk of the dentine has been replaced with a bone-like material with canalicular structure. Although some red blood cells are evident near the deeper channel, no inflammatory cells can be seen.

(Haematoxylin-eosin stain; original magnification $\mathrm{x} 50$.

Reproduced from Heithersay GS, Quintessence Int 1999;30:27-37, courtesy Quintessence International.

present, elective pulpectomy was carried out to allow access to the more deeply infiltrating tissue encircling the root canal (Fig 27d). The canal was prepared with hand instruments and then enlarged with GatesGlidden drills particularly in the coronal third of the root canal to engage the encircling resorptive tissue. Further application of trichloroacetic acid and curettage allowed complete visualization of the resorption defect with the aid of magnification and a focused helium light source (Fig 27e). The canal was then dressed with a corticosteroid/antibiotic paste (Ledermix paste), a therapeutic combination which has been shown to exhibit anti-clastic activity ${ }^{33}$ and the defect was temporarily restored with Cavit (3M/ESPE, Seefeld, Germany). At a subsequent appointment, 18 days later, the temporary filling and intra-canal dressing material were removed by irrigation and sonication. After the canal had been dried, careful inspection with enhanced vision did not reveal any sign of further vascular channels, the resorption cavity was well defined and the adjacent soft tissues showed satisfactory healing (Fig 27f). The canal was then obturated with gutta-percha and AH26 (Dentsply/DeTrey, Konstanz, Germany). A further brief application of trichloroacetic acid ensured a dry field for the insertion of a glass ionomer cement restoration which was protected with a light-activated unfilled bonding resin (Fig 27g). A postoperative radiograph is shown in Fig $27 \mathrm{~h}$. The patient has been re-examined at regular intervals and
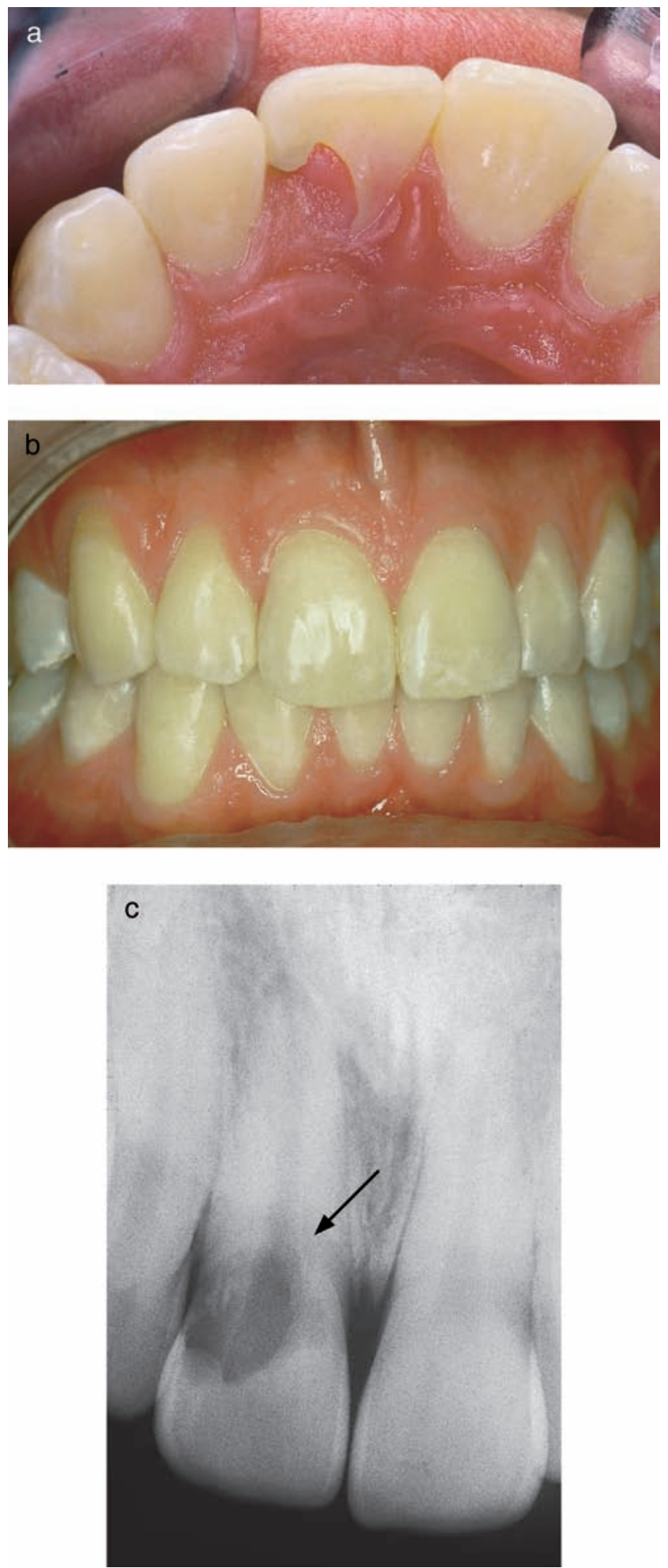

Fig 26a. A mass of soft tissue is evident in a defect on the palatal aspect of the maxillary right central incisor of a 19 year old male with a history of orthodontic treatment 6 years earlier.

Fig 26b. The labial surface of the patient's dentition shows no external sign of the palatal lesion in the maxillary right central incisor.

Fig 26c. The radiograph of the maxillary right central incisor shows a large, irregular radiolucency extending both coronally and into the radicular tooth structure (arrows). This hyperplastic invasive cervical resorptive lesion is classified as Class 3.

Reproduced from Heithersay GS, Quintessence Int 1999;30:96-110, courtesy Quintessence International. 

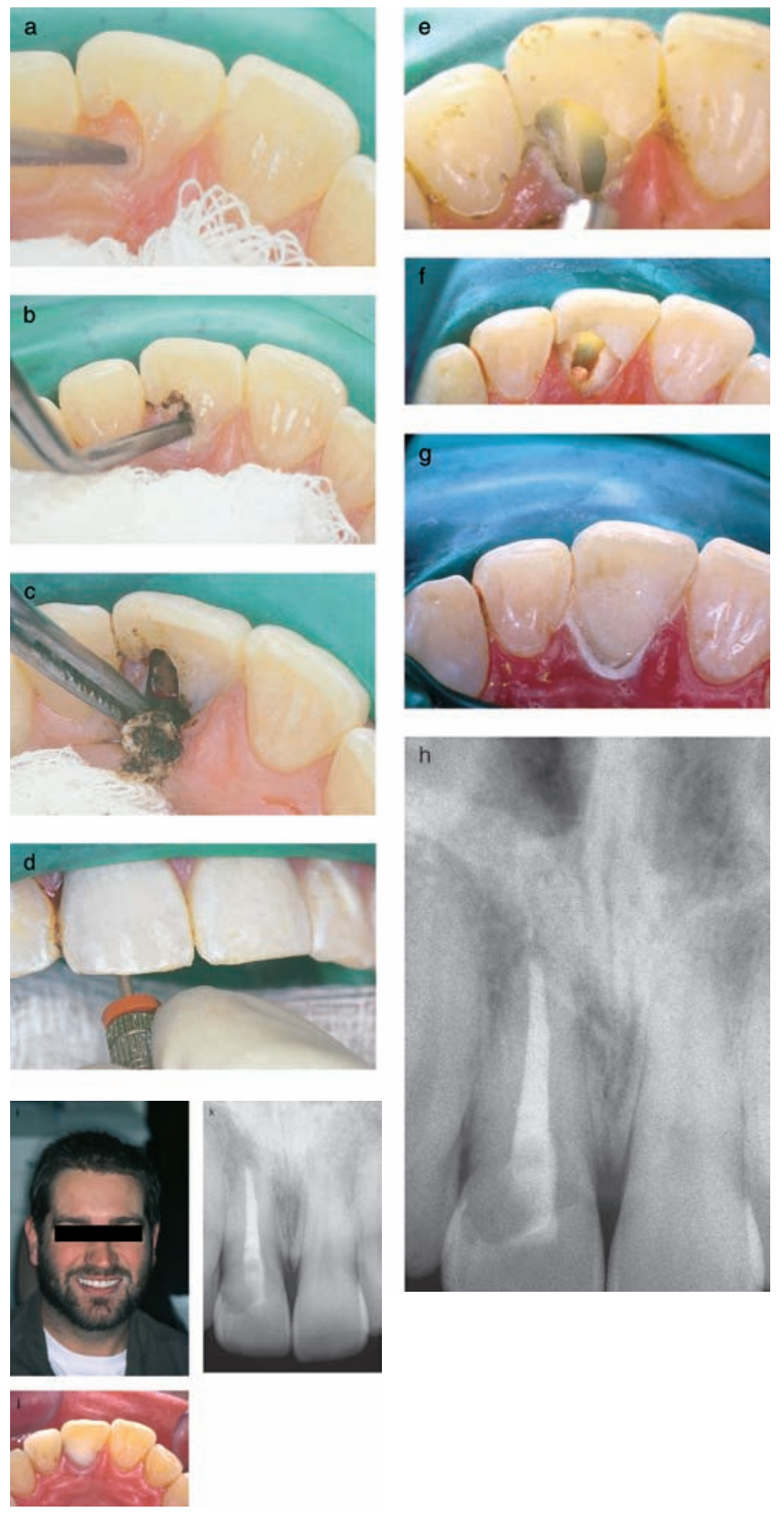

the clinical and radiographic appearance of the tooth 10 years after treatment is shown in Figs 27i-27k.

Orthodontic extrusion can be used to advantage in some Class 3 resorptions by improving access to the base of the resorption cavity and providing a supragingival margin for the restoration. Extrusion is usually effected over 4-6 weeks, using a light wire technique, and followed by splinting, pericision, gingivoplasty and finally restoration.

An internal approach is possible in some Class 3 resorptions but it is essential that the resorptive tissue be traced to the external point (or points) of entry and inactivated by the topical application of trichloroacetic acid prior to the internal placement of a glass-ionomer cement. Alternatively, the defect could be filled with the mineral trioxide aggregate material ProRoot MTA which would appear to possess ideal properties for this type of repair.
Fig 27a. Topical application of trichloracetic acid on a small cotton pellet is carried out with slowly increasing pressure, to prevent haemorrhage.

Fig 27b. Continued application of trichloracetic acid on a small cotton pellet with pressure allows the deeper regions of the lesion to be rendered avascular by the process of coagulation necrosis.

Fig 27c. The affected tissue is curetted from the resorptive cavity to reveal the apparent dentinal base.

Fig 27 d. Elective pulpectomy has been carried out in the central incisor, to allow access to encircling resorptive tissue.

Fig 27e. Following pulpal extirpation, the canal has been enlarged in the coronal third with Gates-Glidden drills to include any resorptive tissue.

Fig 27f. The root canal has been filled with gutta-percha and AH26

18 days after pulpectomy, intracanal dressing with Ledermix paste, and coronal sealing with Cavit. The gingival tissue shows good

healing and the margins of the resorption cavity are well defined.

Fig 27g. A glass-ionomer restoration has been inserted into the resorption cavity following a further topical application of trichloracetic acid to aid in moisture control.

Fig 27h. Postoperative radiograph of the maxillary right central incisor indicates satisfactory filling of the resorptive defect.

Fig $27 \mathrm{i}$. The labial appearance 10 years after treatment shows a satisfactory aesthetic result.

Fig $27 \mathbf{j}$. After 10 years, the palatal gingival tissues appear healthy. The original glass-ionomer restoration has been refaced because of some surface crazing.

Fig 27k. A 10-year follow-up radiograph shows no evidence of further resorption or periradicular pathosis.

Reproduced from Heithersay GS, Quintessence Int 1999;30:96-110, courtesy Quintessence International.

Class 4 resorptions pose far greater difficulties in treatment using this protocol and the poor success rates which have been reported strongly suggest that alternative prosthodontic replacement is generally the preferred clinical option. However, there are occasions when treatment may be justified, providing it does not compromise supporting bone. Orthodontic extrusion is invariably required as an adjunctive treatment if a successful result is to be achieved. Another option in some cases of Class 4 invasive cervical resorption is to leave the affected tooth untreated. However, this may put at risk the health of the supporting bone as a site for implant placement should superimposed periodontal infection develop. The rate of resorption in Class 4 cases has not been investigated but clinical observations suggest that in the absence of superimposed infection, the progress in older patients is slow.

Surgical management: Surgical treatment of varying degrees or invasive cervical treatment has generally involved periodontal flap reflection, curettage, restoration of the defect with amalgam, composite resin or glass-ionomer cement and repositioning the flap to its original position. ${ }^{34-36}$ Periodontal re-attachment cannot be expected with amalgam or composite resin, and is unlikely with glass-ionomer cement, but there is evidence to suggest that this might be possible should ProRoot MTA be used in this situation. ${ }^{37}$ An alternative surgical option is to apically position the flap to the base of the resorption repair. However, should this prove aesthetically unacceptable, orthodontic extrusion can be utilized to improve the gingival contour. 
Table 1.

\begin{tabular}{|c|c|c|}
\hline & & Management \\
\hline \multirow[t]{6}{*}{ Trauma induced } & Surface & Monitor radiographically. Endodontic treatment only if signs of infection. \\
\hline & Transient apical internal & $\begin{array}{l}\text { Monitor radiographically. Endodontic treatment only if signs of infection } \\
\text { or ongoing discolouration. }\end{array}$ \\
\hline & Pressure & Remove cause, e.g., unerupted cuspid, neoplasm. \\
\hline & Orthodontic & Should stabilize on completion of orthodontic treatment. \\
\hline & Replacement & $\begin{array}{l}\text { Mature tooth in normal occlusion; leave and monitor for ultimate implant } \\
\text { replacement. In infra-occlusion; in selected cases surgically reposition, and } \\
\text { treat root surface with Emdogain. }\end{array}$ \\
\hline & & $\begin{array}{l}\text { Immature tooth in infra-occlusion; in selected cases surgically reposition } \\
\text { and treat root surface with Emdogain,or decoronate and submerge. } \\
\text { Implant therapy, if necessary, when alveolar growth completed. }\end{array}$ \\
\hline \multirow[t]{4}{*}{ Infection induced } & Internal inflammatory & $\begin{array}{l}\text { Apical: Endodontic treatment to level of resorption. Long-term calcium } \\
\text { hydroxide dressing before placement of root filling }\end{array}$ \\
\hline & & $\begin{array}{l}\text { Intra-radicular: Endodontic treatment and root canal filling (hot GP } \\
\text { technique, Obtura etc.). }\end{array}$ \\
\hline & & $\begin{array}{l}\text { Prevention; following replantation of mature tooth- pulp extirpation and } \\
\text { Ledermix paste dressing as soon as possible. }\end{array}$ \\
\hline & Communicating internal external & $\begin{array}{l}\text { Endodontic treatment to resorptive defect. Induce calcification by use of } \\
\text { calcium hydroxide alone or following careful topical application of } 90 \% \\
\text { trichloracetic acid. ProRoot MTA may also be used. }\end{array}$ \\
\hline \multirow[t]{7}{*}{ Hyperplastic } & Internal (invasive) replacement & Pulpectomy and root filling. \\
\hline & Invasive coronal & $\begin{array}{l}\text { Carefully apply } 90 \% \text { trichloracetic acid to resorptive tissue after } \\
\text { protecting adjacent soft tissues with glycerol. }\end{array}$ \\
\hline & & $\begin{array}{l}\text { Curette TCA affected resorptive tissue from defect. If pulp involvement, } \\
\text { pulpectomy and root filling after intra-canal dressing with Ledermix paste. } \\
\text { Orthodontic extrusion if necessary. }\end{array}$ \\
\hline & Invasive cervical & $\begin{array}{l}\text { Class } 1,2 . \text { Topical application of } 90 \% \text { trichloracetic acid, curettage, and } \\
\text { glass ionomer cement restoration. }\end{array}$ \\
\hline & & $\begin{array}{l}\text { Class } 3 \text {. Topical application of } 90 \% \text { trichloracetic acid to resorptive tissue, } \\
\text { curettage, elective pulpectomy and canal preparation to gain access to } \\
\text { deeper and encircling infiltrative channels. Ledermix paste intra-canal } \\
\text { dressing, followed by root filling and final glass ionomer cement } \\
\text { restoration. Adjunctive orthodontic extrusion if necessary. }\end{array}$ \\
\hline & & $\begin{array}{l}\text { Alternative therapy - periodontal flap reflection, curettage, TCA } \\
\text { application to the defect, endododontic therapy and restoration. }\end{array}$ \\
\hline & & Class 4. Leave untreated and monitor or extract and implant. \\
\hline
\end{tabular}

\section{CONCLUSION}

An alternative classification of dental resorptions based on aetiological groups has been used to define dental resorption and then to apply management to each group and sub-groups. The groups are: (1) trauma induced dental resorption; (2) infection induced dental resorption; and (3) hyperplastic invasive dental resorptions. The diagnosis of dental resorptions and an understanding of the underlying pathosis within each group is critical to clinical management. A summary of the groups and sub-groups of dental resorptions and their management is shown in Table 1.

\section{ACKNOWLEDGEMENTS}

The author wishes to acknowledge Quintessence International for their permission to reproduce figures from his original publications. Professor Sven Lindskog and Dr John McNamara also kindly allowed the reproduction of some of their research and clinical material. In addition, he wishes to acknowledge the assistance of Dr William Kahler and Dr Khashnary Nassery in the preparation of this paper.

\section{REFERENCES}

1. Pierce A. Pathophysiological and therapeutic aspects of dentoalveolar resorption. Aust Dent J 1989;34:437-448.

2. Lindskog SF, Dreyer CW, Pierce AM. Osteoclastic activity. In: Andreasen JO, Andreasen FM, Andersson L, eds. Textbook and color atlas of traumatic injuries of the teeth. 4th edn. Blackwell Munksgaard, 2006.

3. Davidovitch Z. Biological mechanisms of tooth eruption, resorption and replacement by implants. Birmingham, Alabama: EBSCO Media, 1994.

4. Tronstad L. Root resorption - etiology, terminology and clinical manifestations. Endod Dent Traumatol 1988;4:241-252.

5. Trope M. Root resorption due to dental trauma. Endod Topics 2002;1:79-100.

6. Andreasen JO, Andreasen FM, Andersson L, eds. Textbook and color atlas of traumatic injuries to the teeth. 4th edn. Blackwell Munksgaard, 2006.

7. Andreasen JO, Andreasen FM, Blakland LK, Flores MT, eds. Traumatic dental injuries; a manual. 2nd edn. Blackwell Munksgaard, 2004.

8. Andreasen JO. Luxation of permanent teeth due to trauma. A clinical and radiographic follow-up study of 189 injured teeth. Scand J Dental Res 1970; 78:273-286.

9. Andreasen FM. Transient apical breakdown and its relation to color and sensibility changes after luxation injuries to teeth. Endod Dent Traumatol 1986;2:9-19. 
10. Heithersay GS. Invasive cervical resorption. Endod Topics 2004;7:73-92.

11. Heithersay GS Invasive cervical resorption: An analysis of potential predisposing factors. Quintessence Int 1999;30:83-95.

12. Lindskog S, Heithersay GS, Pierce AM. Dental resorptions. In: Scandinavian Yearbook of Dentistry. Blackwell Munksgaard, 2006 (in press).

13. Malmgrem B. Decoronation of an ankylosed tooth for preservation of alveolar bone prior to implant placement. Dent Traumatol 2001;17:93-95.

14. Koh ET, Torabinejad M, Pitt-Ford TR, Brady K, McDonald F. Mineral trioxide aggregrate stimulates a biological response in human osteoblasts. J Biomed Mater Res 1997;5:432-439.

15. Vier FV, Figueiredo JA. Internal apical resorption and its correlation with the type of apical lesion. Int Endod J 2004;37:730-737.

16. Buchanan S. ProSystem GT: design, technique, and advantages. Endod Topics 2005;10:168-175.

17. Camilleri J, Pitt Ford TR. Mineral trioxide aggregate: a review of the constituents and biological properties of the material. Int Endod J 2006;39:747-754.

18. Goldberg F, Massone EJ, Esmoris M, Alfie D. Comparison of different techniques for obturating experimental internal resorptive cavities. Endod Dent Traumatol 2000;16:116-121.

19. Abbott PV, Heijkoop PS, Cardaci SC, Heithersay GS, Hume WR. An SEM study of the effects of different irrigation sequences and utrasonics. Int Endod J 1991;24:308-316.

20. Abbott PV, Heithersay GS, Hume WR. The release and diffusion through human coronal dentine in vitro of triamcinolone and demeclocycline from Ledermix Paste. Endod Dent Traumatol 1989;5:92-97.

21. Pierce A, Lindskog S. The effect of an antibiotic/cortico-steriod paste on inflammatory root resorption in vivo. Oral Surg Oral Med Oral Pathol 1987;64:216-220.

22. Cvek M. Endodontic management of traumatised teeth. In: Andreasen JO, Andreasen FM, eds. Textbook and color atlas of traumatic injuries to the teeth. 3rd edn. Copenhagen: Munskgaard, 1994:560-561.

23. Bryson EC, Levin L, Branchs F, Abbott PV, Trope M. Effect of immediate intra-canal placement of Ledermix Paste ${ }^{\circledR}$ on healing of replanted dog teeth after extended dry times. Dent Traumatol 2002;18:316-321.

24. Andreasen JO. The effect of pulp extirpation or root canal treatment on periodontal healing after replantation of mature permanent incisors in monkeys. J Endod 1981;7:245-252.

25. Lengheden A, Blomlöf L, Lindskog S. Effect of immediate calcium hydroxide treatment and permanent root-filling on periodontal healing in contaminated replanted teeth. Scand J Dent Res 1991;99:139-146.
26. Lengheden A, Blomlöf L, Lindskog S. Effect of delayed calcium hydroxide treatment on periodontal healing in contaminated replanted teeth. Scand J Dent Res 1991; 99:147-153.

27. Wade AB. Basic Periodontology. Bristol, England: Wright \& Sons, 1960:156-159.

28. Heithersay GS. Clinical, radiologic and histopathologic features of invasive cervical resorption. Quintessence Int 1999;30:27-37.

29. Wedenberg C. Development and morphology of internal resorption in teeth - A study in humans, monkeys and rats. Stockholm, Sweden: Karolinska Institute, 1987. PhD thesis.

30. Heithersay GS. Treatment of invasive cervical resorption: An analysis of results using topical application of trichloroacetic acid, curettage, and restoration. Quintessence Int 1999;30:96110.

31. Gaskill JH. Report of a case of internal resorption. Dental Cosmos 1894;36:1019-1024.

32. Mummery JH. The pathology of "pink spots" on teeth. Br Dent J 1920;41:301-311.

33. Pierce A, Lindskog S. The effect of an antibiotic/corticosteriod paste on inflammatory root resorption in vivo. Oral Surg Oral Med Oral Pathol 1987;64:216-220.

34. Lustman J, Ehrlich J. Deep external resorption: Treatment by combined endodontic and surgical approach. A report of 2 cases. Int Dent J 1974;24:203-206.

35. Goodman JR, Wolfe GN. The treatment of cervical external resorption in adolescents. Br Dent J 1980;149:234-236.

36. Trope M. Subattachment inflammatory root resorption: Treatment strategies. Pract Periodont Aesthet Dent 1998;10:1005-1010

37. Pitt Ford TR, Torabinejad M, McHendry DJ, Hong CU, Kariyawasam SP. Use of mineral trioxide aggregate for repair of furcal perforations. Oral Surg Oral Med Oral Pathol 1995;79:756-763.

Address for correspondence/reprints. Professor GS Heithersay AO School of Dentistry Faculty of Health Sciences The University of Adelaide Adelaide, South Australia 5005 Email: geoffrey.heithersay@adelaide.edu.au 